\title{
$\alpha$-Spectrin and integrins act together to regulate actomyosin and columnarization, and to maintain a monolayered follicular epithelium
}

\author{
Bing Fu Ng ${ }^{1, \mp}$, Gokul Kannan Selvaraj ${ }^{1, \ddagger}$, Carmen Santa-Cruz Mateos ${ }^{2}$, Inna Grosheva ${ }^{2}$, Ines Alvarez-Garcia ${ }^{1, *}$, \\ María Dolores Martín-Bermudo ${ }^{2}$ and Isabel M. Palacios ${ }^{1, \S}$
}

\begin{abstract}
The spectrin cytoskeleton crosslinks actin to the membrane, and although it has been greatly studied in erythrocytes, much is unknown about its function in epithelia. We have studied the role of spectrins during epithelia morphogenesis using the Drosophila follicular epithelium (FE). As previously described, we show that $\alpha$-Spectrin and $\beta$-Spectrin are essential to maintain a monolayered FE, but, contrary to previous work, spectrins are not required to control proliferation. Furthermore, spectrin mutant cells show differentiation and polarity defects only in the ectopic layers of stratified epithelia, similar to integrin mutants. Our results identify $\alpha$-Spectrin and integrins as novel regulators of apical constriction-independent cell elongation, as $\alpha$-Spectrin and integrin mutant cells fail to columnarize. Finally, we show that increasing and reducing the activity of the Rho1Myosin II pathway enhances and decreases multilayering of $\alpha$ Spectrin cells, respectively. Similarly, higher Myosin II activity enhances the integrin multilayering phenotype. This work identifies a primary role for $\alpha$-Spectrin in controlling cell shape, perhaps by modulating actomyosin. In summary, we suggest that a functional spectrin-integrin complex is essential to balance adequate forces, in order to maintain a monolayered epithelium.
\end{abstract}

KEY WORDS: Tissue architecture, Epithelium, Monolayer, Tumorlike mass, Proliferation, Cell shape

\section{INTRODUCTION}

Monolayered epithelia are sheets of adherent, polarized cells that act as physical barriers and constitute structural components of organs and tissues. The formation and maintenance of the monolayered structure are crucial for both proper function of the epithelia and whole-body homeostasis. During carcinogenesis, loss of epithelial architecture leads to the formation of multilayered epithelia, disorganized cell masses and increased tumorigenic potential.

The Drosophila melanogaster ovary constitutes an excellent model system in which to study the molecular and cellular basis of epithelial morphogenesis. The adult ovary is composed of various

\footnotetext{
${ }^{1}$ Department of Zoology, University of Cambridge, Downing Street, Cambridge CB2 3EJ, UK. ${ }^{2}$ Centro Andaluz de Biología del Desarrollo CSIC-Univ. Pablo de Olavide, Sevilla 41013, Spain.

*Present address: PLOS Biology, Carlyle Road, Cambridge CB4 3DN, UK

tThese authors contributed equally to this work

§Author for correspondence (mip22@cam.ac.uk)
}

This is an Open Access article distributed under the terms of the Creative Commons Attribution License (http://creativecommons.org/licenses/by/3.0), which permits unrestricted use, distribution and reproduction in any medium provided that the original work is properly attributed.

Received 20 August 2015; Accepted 18 February 2016 ovarioles that contain a line of egg chambers at different developmental stages [stage1-14 (S1-14)]. Each egg chamber is composed of 16 germline cells (including the oocyte), and a layer of somatic cells (the follicle cells, FCs) forming a monolayered epithelium termed the follicular epithelium (FE) (Fig. 1A). FCs are derived from stem cells that are located in the germarium. Up to S6 of oogenesis, FCs undergo several rounds of mitotic cycles to form the FE, then exit mitosis and enter an endocycle. From S7, most FCs change their shape from cuboidal to columnar, and migrate towards the posterior (Fig. S1). The factors important for formation of a monolayered FE are not yet fully understood, but mutations in genes controlling polarity and mitosis lead to FE multilayering, such as aPKC (Abdelilah-Seyfried et al., 2003), Notch and the Hippo pathway (Meignin et al., 2007; Polesello and Tapon, 2007; Yu et al., 2008). In addition, integrins and spectrins (Spec) are also important for maintaining a monolayer.

The spectrin-based membrane skeleton (SBMS) is a scaffold made from building blocks of tetramers of two $\alpha$ and two $\beta$ Spec subunits that line the cell membrane. The function of the SBMS has been greatly studied in erythroid cells, and a variety of erythrocyte disorders are associated with mutations in Spec genes. Members of the Spec family are conserved in all eukaryotes, with a greater conservation between Drosophila and mammalian non-erythroid Specs than between erythroid and non-erythroid mammalian forms (Baines, 2003, 2009; Salomao et al., 2006). However, in contrast to mammals, the Drosophila genome features a single form of $\alpha$-Spec (human $\alpha I I-l i k e)$, a conventional $\beta$ subunit (human $\beta I I-l i k e)$ and a heavy $\beta$ subunit $\left(\beta_{\mathrm{H}}\right)$, making it easier to characterize their function in non-erythroid cells (Byers et al., 1989; Dubreuil et al., 1990; Lee et al., 1997). The $(\alpha \beta)_{2}$ and $\left(\alpha \beta_{\mathrm{H}}\right)_{2}$ tetramers are distinctively localized in the basolateral and apical domains, respectively (Dubreuil et al., 1998; Lee et al., 1997; Thomas et al., 1998; Zarnescu and Thomas, 1999).

A diversity of functions has been attributed to spectrins based on studies in both cell culture and model organisms. In invertebrates, spectrins are essential for morphogenesis and animal growth. Drosophila spectrins have been recently identified as modulators of the cell growth Hippo pathway in various tissues (Deng et al., 2015; Fletcher et al., 2015; Wong et al., 2015). In ovaries, a mutant $\beta$-Spec allele with a premature stop codon at amino-acid 1046 shows defects in FE integrity, actin organization and oocyte polarity, partially phenocopying hippo mutants (Wong et al., 2015). $\alpha$-Spec mutant FCs also form a stratified epithelium, with polarity defects (Lee et al., 1997), and FCs expressing an $\alpha$-Spec RNAi show hippo-like differentiation defects (Fletcher et al., 2015). By contrast, $\beta_{H}$-Spec does not regulate Hippo or actin in ovaries (Fletcher et al., 2015; Thomas et al., 1998; Zarnescu and Thomas, 1999). These findings confirm the idea that different tissues exhibit different dependence 
A
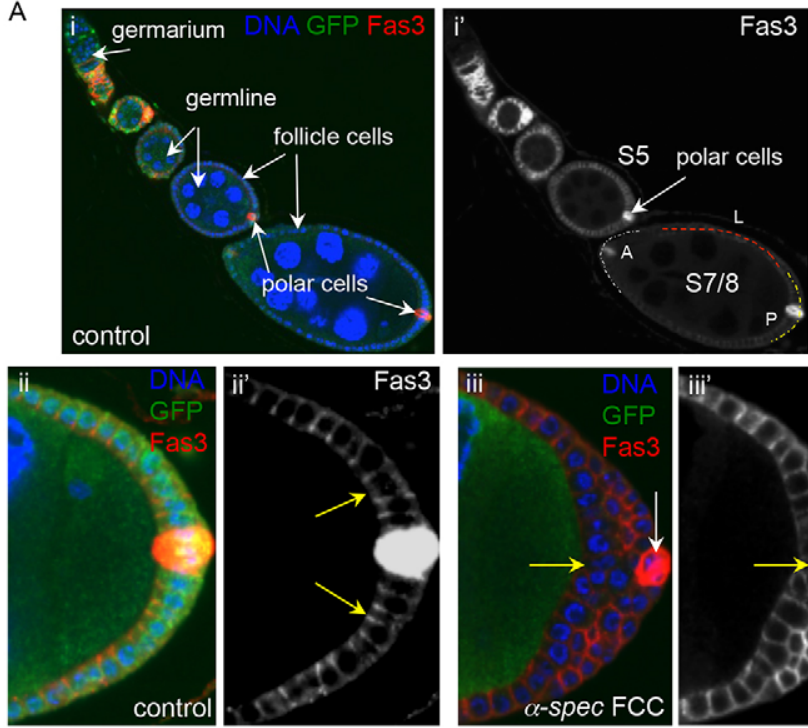

B

Stage 9 egg chambers

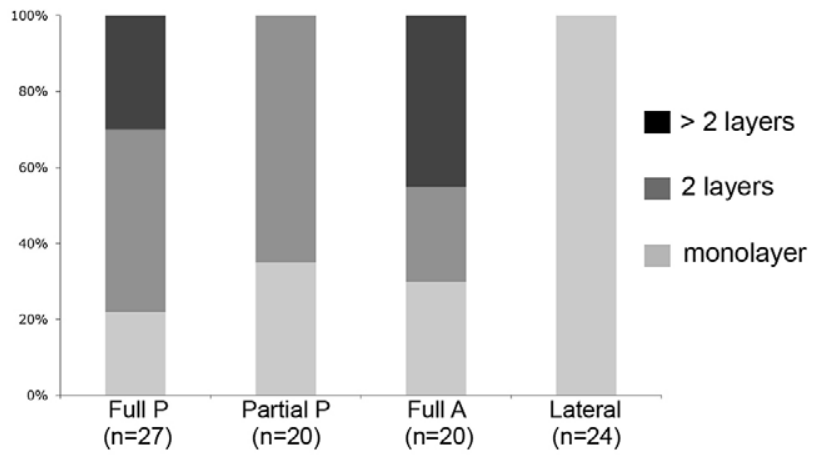

C
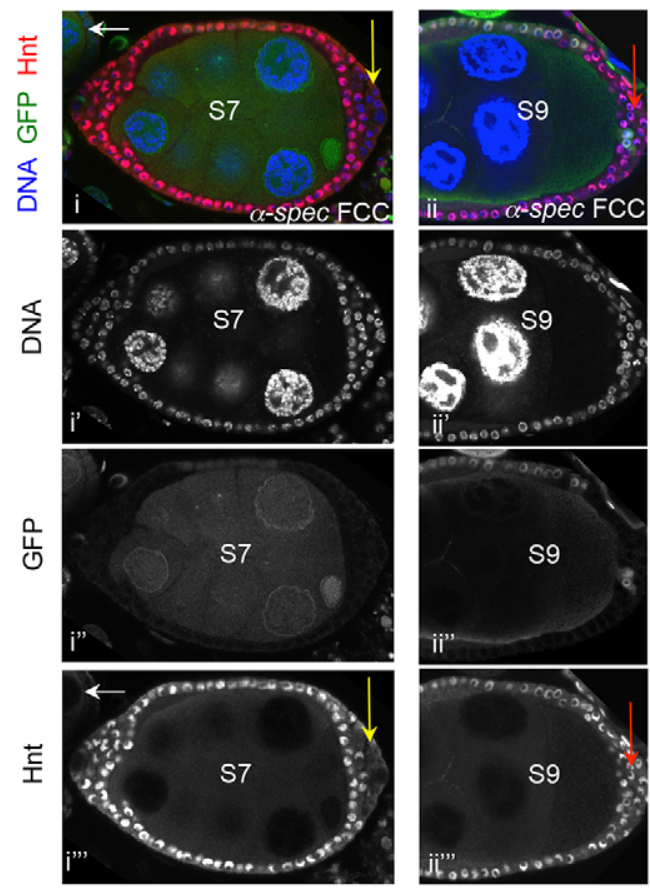

Hnt expression

$\square$ No defect $\mathbf{\square} 1-2$ cells defective $\square \geq 3$ cells defective

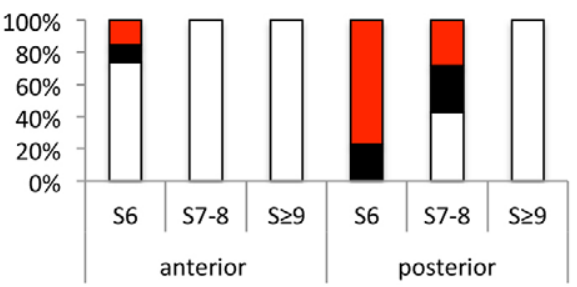

Fig. 1. $\alpha$-Spectrin mutant epithelia form multilayers with aberrant Hindsight and Fasciclin 3 expression only in ectopic layers. (A) Fas 3 is expressed in the FE in early oogenesis (i,i', egg chambers budding off of the germarium), but becomes restricted to the polar cells by S5 (i',ii). When $\alpha$-Spec cells (follicle cell clones, FCCs) form a multilayer (iii), cells of the ectopic layers express a Fas3 level intermediate to those of the germline-adjoining FCs (yellow arrow) and the polar cells (white arrows) ( $n=15)$. Note in ii the accumulation of Fas 3 in an apicolateral region (yellow arrows in ii'), which is relevant for later findings. (i') From S7, the epithelium is divided into lateral (L, red line), anterior ( $\mathrm{A}$, white line) and posterior (P, yellow line) domains. The posterior FC (PFC) domain is formed by a cap of $\sim 200$ cells surrounding the polar cells, and correlates in the cross-sections presented in most figures with a region that expands by ten cells to each side of the polar cells. Panels ii-iii' show the posterior domain. Fas3 is in red in merge panels. (B) Quantification reveals that epithelial stratification is never observed in $\alpha$-Spec mutant lateral epithelia (Lateral), being limited to the terminal (anterior, A, or posterior, P) domains only. Epithelial integrity is compromised even when $\alpha$-Spec clones make up part of the posterior (Partial P clone). (C) Hnt is not expressed before S6 of oogenesis (white arrows in i and i"'). After S6 the Notch pathway activates Hnt expression. This Notch-dependent upregulation of Hnt is often defective in the ectopic layers of S6-8 $\alpha$-Spec multilayered epithelia (yellow arrows in i and $\mathrm{i}^{\prime \prime \prime}$ ). However, Hnt upregulation is normal by S9, even in ectopic layers (red arrows in ii and ii"'; see also D). The egg chambers are positioned with the anterior to the left. Hnt is in red in merge panels. (D) Quantification of mosaic egg chambers containing control and $\alpha$-Spec mutant cells reveals that young egg chambers (S6-8) have severe Hnt expression defects (at least three cells defective), whereas older egg chambers (S9 and beyond) are non-defective. This trend is more pronounced at the posterior. $n=45,32$ and 24 for S6, S7-8 and S $\geq 9$, respectively. In A and C, merged images show DAPI in blue, and mutant cells lack GFP.

on the apical versus lateral spectrin cytoskeleton (Dubreuil et al., 2000; Hulsmeier et al., 2007; Thomas and Williams, 1999).

These previous studies concentrated on spectrins as Hippo modulators. We decided to broaden the analysis of SBMS function in a monolayered epithelium by studying the consequences of eliminating SBMS on the proliferation, polarization and differentiation of FCs, as well as on the architecture of the FE. As $\alpha$-Spec is the major component of both the apical and lateral spectrin cytoskeletons, we decided to concentrate on $\alpha$-Spec. Eliminating $\alpha$-Spec in the FE results in stratification of the terminal regions, especially the posterior, supporting previous findings (Fletcher et al., 2015; Lee et al., 1997). However, in contrast to Hippo, we find that the function of the SBMS in the monolayered FE is not to control mitosis, differentiation or polarity, but to regulate the actomyosin cytoskeleton, septate junctions (SJs) and cell shape. The $\alpha$-Spec mutant phenotype is similar to that of integrin [myospheroid (mys)] mutants, and $\alpha$-Spec and integrins colocalize in the lateral membrane of the FCs. We propose that a functional spectrin-integrin complex is important for regulation of the actomyosin cytoskeleton and tissue architecture.

\section{RESULTS}

$\alpha$-Spectrin mutant cells show differentiation defects only when forming a stratified epithelium

To understand the defects of eliminating $\alpha$-Spec on FE morphogenesis, we studied FCs mutant for the null allele $\alpha$-Spec ${ }^{\text {rg41 }}$. As in previous reports, we found that $\alpha$-Spec FCs 
form a multilayered epithelium. These tumor-like masses are observed only at the terminal (anterior/posterior) domains of the egg chamber, and never at the mid-body (Fig. 1). This terminal requirement for $\alpha$-Spec in epithelial architecture is identical to the terminal requirement for Hippo and integrins (Fernández-Miñán et al., 2007; Meignin et al., 2007; Polesello and Tapon, 2007).

Mutations that result in a multilayered FE often prevent the FCs from differentiating. FCs differentiate from $\mathrm{S} 6$, as revealed by differential expression of markers, such as Fasciclin 3 (Fas3), Hindsight (Hnt; also known as Pebbled) and Eyes absent (Eya). Fas3 is expressed at high levels in immature FCs, but its expression becomes gradually restricted to the polar cells as oogenesis proceeds (Bai, 2002; Muzzopappa and Wappner, 2005) (Fig. 1Ai), whereas Hnt is upregulated from S6 (Sun and Deng, 2007). Analysis of mosaic FE containing both control and $\alpha$-Spec mutant FCs (also named FCCs) revealed that some S6-8 $\alpha$-Spec FCCs show high levels of Fas 3 (Fig. 1Ai-Aii versus Aiii) and lack Hnt expression (Fig. 1Ci,D). However, in all cases, the defects are only observed in $\alpha$-Spec cells located in the ectopic layers of the multilayered epithelium (Fig. 1A,C), and not in mutant cells that are either adjacent to the oocyte (Fig. 1Aiii, yellow arrow) or forming a monolayer (Fig. 5). This differentiation phenotype is stronger at the posterior than at the anterior pole, and weaker in older egg chambers: $100 \%(n=45)$ of the S6 $\alpha$-Spec FCCs show Hnt defects, but these defects are absent at S9 $(n=24)$ (Fig. 1C,D). Similarly, fewer $\alpha$-Spec FCCs have defective Fas3 levels in older egg chambers $(62.5 \%$ and $25 \%$ in early S6-8 and S9 egg chambers, respectively, $n=16$ ). It is interesting to note that even though Fas 3 is properly downregulated in most $\alpha$-Spec cells, the remaining Fas 3 does not show the apicolateral accumulation that is observed in control posterior FCs (PFCs; Fig. 1Aii' versus Aiii", yellow arrows; Fig. 5; Dubreuil et al., 2001). Finally, Eya, which is downregulated in cells from S6, was also properly downregulated in $\alpha$-Spec mutant monolayers, and $\alpha$-Spec cells adjacent to the oocyte, but had a stronger expression in the $\alpha$-Spec ectopic layers (data not shown).

Thus, $\alpha-S p e c$ is required for the FE to maintain a monolayer, and for the FCs to mature only when part of ectopic layers. These varying defects in differentiation indicate that $\alpha$-Spec plays a secondary role in this aspect of oogenesis, unlike the Hippo pathway, which fully blocks FC maturation when mutated in terminal FCs (Meignin et al., 2007; Polesello and Tapon, 2007).

\section{$\alpha$-Spectrin mutant cells located in the ectopic layers show polarity defects}

Recent work has shown that apical and lateral components [such as $\alpha$-Spec, aPKC and Discs large (Dlg; Dlg1 - FlyBase)] localize properly in $\beta$-Spec mutant monolayers (Wong et al., 2015). By contrast, $\alpha$-Spec FCs that are part of a hyperplastic region show a loss of epithelial polarity (Lee et al., 1997). However, the $\alpha$-Spec ovaries analyzed by Lee et al. were also heterozygous for discs lost $(d l t)$, a polarity gene. The $d l t$ heterozygosity might have increased the polarity phenotype in $\alpha$-Spec mutant FCs. To characterize further the role of $\alpha$-Spec in epithelial polarity, we analyzed the distribution of aPKC and Dlg in FCs that lack only $\alpha$-Spec. These proteins localize correctly in $\alpha$-Spec FCCs that maintain a monolayered epithelium (Fig. 2A; $n=15$ ). However, mutant cells in ectopic layers show mislocalized aPKC and Dlg in $80 \%$ and $97 \%$ of the mutant epithelia, respectively (Fig. $2 \mathrm{~B} ; n=35$ ). aPKC often expands into the lateral membrane (red arrows) and by S8-9 Dlg is no longer found restricted to the lateral membrane (yellow arrows), resulting in the colocalization of these two proteins in some instances (Fig. 2Bi, white arrows). However, some degree of
A

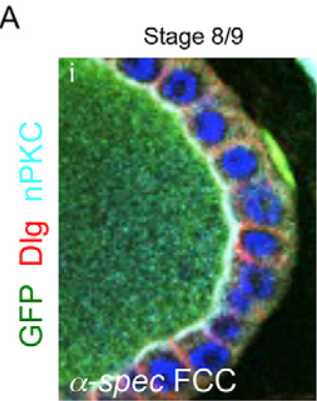

B
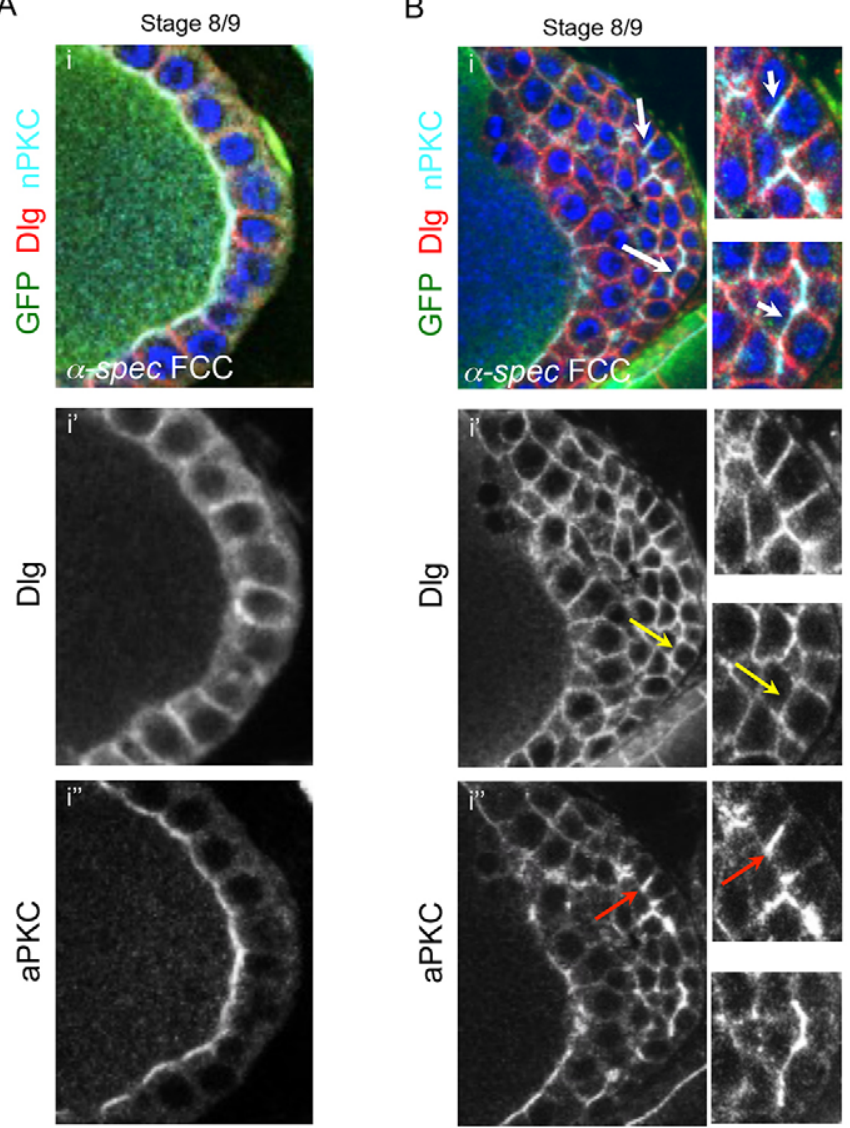

Fig. 2. $\alpha$-Spectrin mutant follicle cells display epithelial polarity defects in ectopic layers. (A) The lateral marker DIg and the apical marker aPKC localize correctly in $\alpha$-Spec mutant cells that form a monolayer $(n=12)$. (B) In a multilayered $\alpha$-Spec mutant FE, correct localization of Dlg and aPKC is observed in germline-contacting FCs. In ectopic layers, Dlg is often mislocalized between ectopic layers of cells (i', yellow arrows), and aPKC often expands into the lateral membrane ( $\mathrm{i}^{\prime \prime}$, red arrows). The white arrows in the top panel indicate colocalization between the mislocalized markers. Some degree of polarity is still preserved because Dlg and aPKC are generally localized correctly in $\alpha$-Spec ectopic layers $(n=35)$. All images show the posterior domain of S8/9 egg chambers. Blue, DAPI; red, Dlg; light blue, aPKC. $\alpha$-Spec mutant clones (FCCs) lack GFP.

polarity is still preserved, as Dlg and aPKC can localize correctly in the most ectopic layer.

All these observations suggest that spectrins become relevant for epithelial polarity when FCs lose contact with the germline or the basal membrane, or that an apical and basal cue can compensate for the lack of a spectrin cytoskeleton.

\section{Oocyte polarity is largely unaffected in egg chambers with $\alpha$-Spectrin mutant follicle cells}

The repolarization of the oocyte in mid-oogenesis is induced by a signal from the PFCs (Deng and Ruohola-Baker, 2000; Frydman and Spradling, 2001; González-Reyes et al., 1995; MacDougall et al., 2001; Roth et al., 1995), a process suggested to depend on the SBMS, as oocyte polarity is often aberrant in egg chambers with $\beta$-Spec clones (Wong et al., 2015) or $\alpha$-Spec, $d l t /+$ cells (Lee et al., 1997). As we have observed that oocyte-adjacent $\alpha$-Spec cells mature and polarize properly, we wondered whether elimination of only $\alpha$-Spec affects repolarization of the oocyte. In wild-type egg chambers, oocyte nucleus migration from posterior to the dorsalanterior corner is complete by $\mathrm{S} 7$, and provides a read-out of oocyte 
polarity. This migration is also observed in $95.5 \%$ of egg chambers with large $\alpha$-Spec FCCs (Fig. S2; $n=45$ ). A more sensitive assay to detect oocyte polarity defects is Staufen localization, which by S9 forms a tight crescent at the posterior of the oocyte (Fig. S2A). Of the $95.5 \%$ mutant egg chambers that showed wild-type nucleus positioning, we found that Staufen is localized properly in all of them, whereas $13 \%$ of follicles with partial posterior clones show Staufen expressed in a crescent-shaped area that is shifted towards the control cells (Fig. S2B,D; data not shown). Thus, oocyte polarity is largely unaffected in egg chambers with $\alpha$-Spec mutant PFCs.

\section{$\alpha$-Spectrin is not required for cells to exit mitosis}

The results described above show that $\alpha$-Spec FCCs show polarity and differentiation defects only in the ectopic layers of a multilayered epithelium, and induce mild oocyte polarity defects. By contrast, PFC differentiation and oocyte polarity is aberrant in all hippo mosaic egg chambers (Meignin et al., 2007; Polesello and Tapon, 2007; Yu et al., 2008). To test whether $\alpha$-Spec FCCs show any hippo-like phenotype, we analyzed whether $\alpha$-Spec is required for the FCs to exit mitosis, a process that is Hippo dependent (Meignin et al., 2007), by detecting phospho-histone 3/PH3. PH3 is only detected until S6, and never later, in control cells (Fig. 3A) (Deng et al., 2001; Schaeffer et al., 2004). By contrast, hippo FCs are often positive for PH3 at S7-10B (Fig. 3B,E) (Meignin et al., 2007; Polesello and Tapon, 2007). As with wild type, $\alpha$-Spec FCCs never express PH3 after S6 (Fig. 3C,D). Hence, and unlike Hippo, $\alpha$-Spec is not required for FCs to exit mitosis.

\section{Septate junction-components mislocalize in $\alpha$-Spectrin mutant cells}

Our initial observation that Fas3 accumulates apicolaterally in S9 PFCs (Fig. 1Aii'), and that such localization was lost in $\alpha$-Spec cells in contact with the oocyte (Fig. 1Aiii'), suggest that $\alpha$-Spec and Fas3 might be involved in processes other than FC maturation and polarity (as these processes are not aberrant in oocyte-adjacent $\alpha$ Spec cells). Earlier works identified Fas 3 as a component of SJs, the invertebrate counterparts of tight junctions (TJs) (Brower et al., 1980; Fehon et al., 1994; Patel et al., 1987; Woods and Bryant, 1991). SJs form basal to the zonula adherens (ZA), and in addition to blocking diffusion they also aid cell adhesion (Fehon et al., 1991; Tepass and Tanentzapf, 2001; Woods and Bryant, 1993; Zak and Shilo, 1992). The aberrant distribution of Fas3 in $\alpha$-Spec FCs raises the possibility that $\alpha-S p e c$ is required for SJs formation. Thus, we characterized the localization pattern during oogenesis of the SJ components Fas3, Dlg (Woods et al., 1996), Coracle (Cora) (Fehon et al., 1994) and Fas2 (Wei et al., 2004).

All four proteins are expressed uniformly along the lateral membrane in early FE (Fig. 4A-D). From S7, an apicolateral concentration of these proteins becomes distinct in PFCs. This apicolateral localization has been previously observed for Fas3, and our findings show Dlg, Cora and Fas2 to behave similarly. These observations are consistent with transmission electron micrographs that detected incipient SJs at S6 (Müller, 2000), and suggest that the loss of apicolateral accumulation of Fas 3 in $\alpha$-Spec FCs might be related to defects in $\mathrm{SJ}$ formation. In fact, the apicolateral localization of Fas3, Dlg, Cora and Fas 2 is absent in $\alpha$-Spec cells (Fig. 5), even when the mutant cells form a monolayer. It is unclear at this resolution how the SJ components localize in mutant cells; the proteins sometimes extend along the lateral membrane (white arrows) or are absent altogether (yellow arrows). However, a departure from the normal apicolateral accumulation is clear, and it suggests a function for $\alpha$-Spec in SJ formation.

These localization defects are specific to SJ proteins, and not to all apical junctions, as the adherens junction markers Armadillo
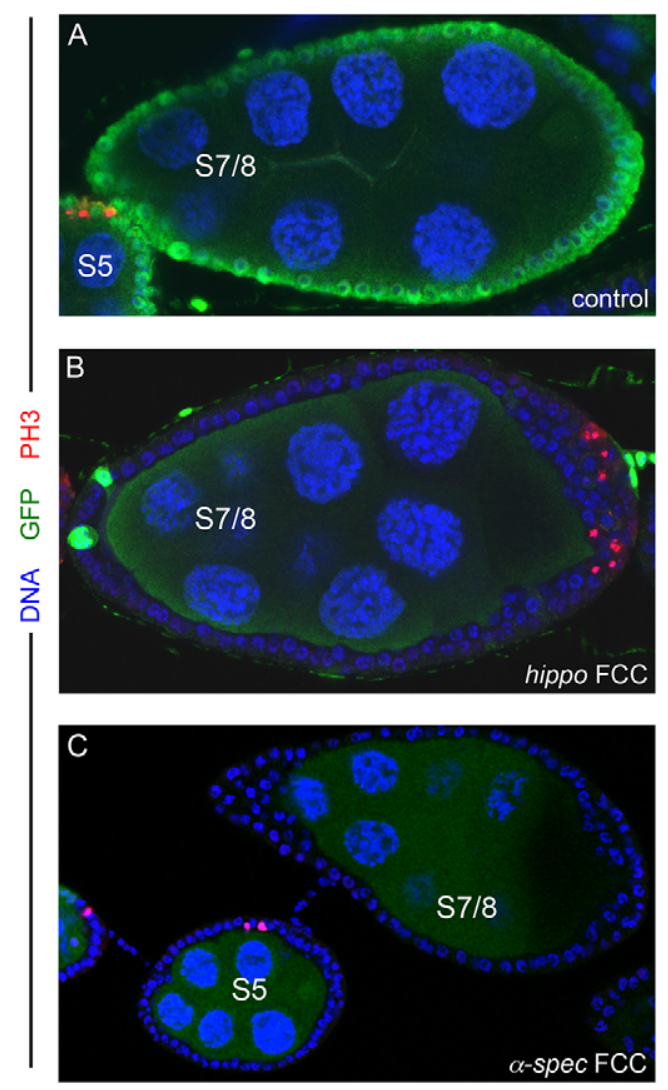
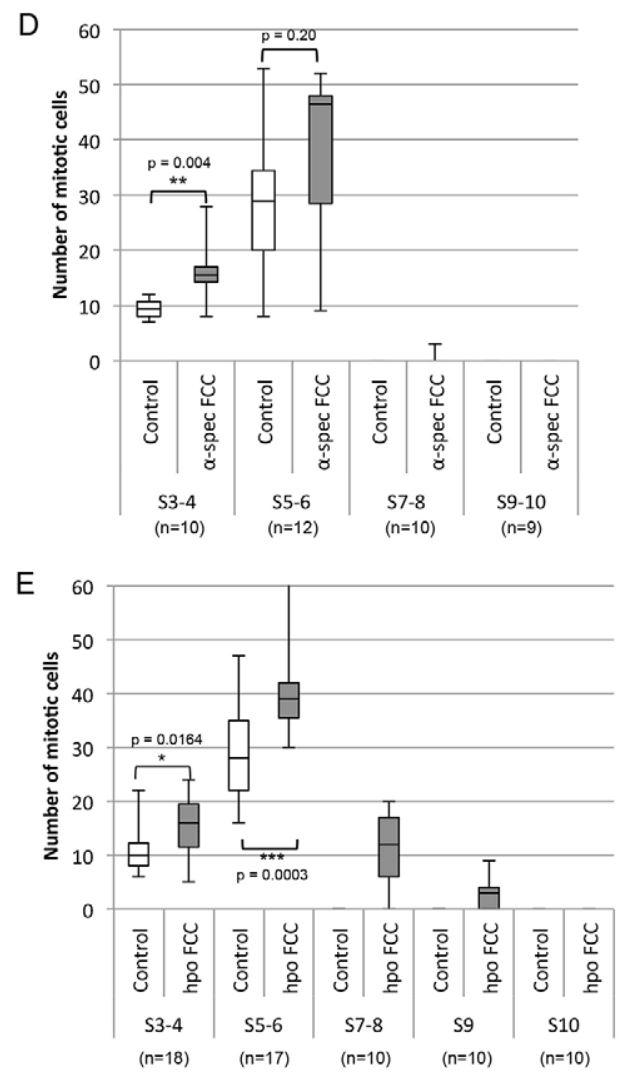

Fig. 3. Unlike the Hippo pathway, $\alpha$-Spectrin is not required for follicle cells to exit mitosis. (A-C) In contrast to hippo mutant cells (hippo FCCs, B), both control (A) and $\alpha$-Spec FCCs (C) exit mitosis at $\mathrm{S} 6$ of oogenesis. (A) Control egg chambers showing $\mathrm{PH} 3$ (red) positive cells at S5, but not at S7/8. (B) S7/8 egg chamber with $\mathrm{PH} 3$ (red) positive hippo cells. (C) As in control, $\alpha$-Spec FCCs are not positive for $\mathrm{PH} 3$ after $\mathrm{S} 6$ of oogenesis. Mutant cells lack GFP. Blue, DAPI. $(\mathrm{D}, \mathrm{E})$ Box plot quantification of mitotic cells (indicated by $\mathrm{PH} 3$ expression) in $\alpha-S p e c$ (D) or hippo (E) S3-S10 egg chambers. S7-9 egg chambers containing hippo FCCs are positive for PH3. $\alpha$-Spec FCCs are not positive for $\mathrm{PH} 3$ after $\mathrm{S} 6$, but they do display higher instances of $\mathrm{PH} 3$ in earlier stages (quantified in S3-6). $n$ is as indicated for both the control and the mutant cells. 


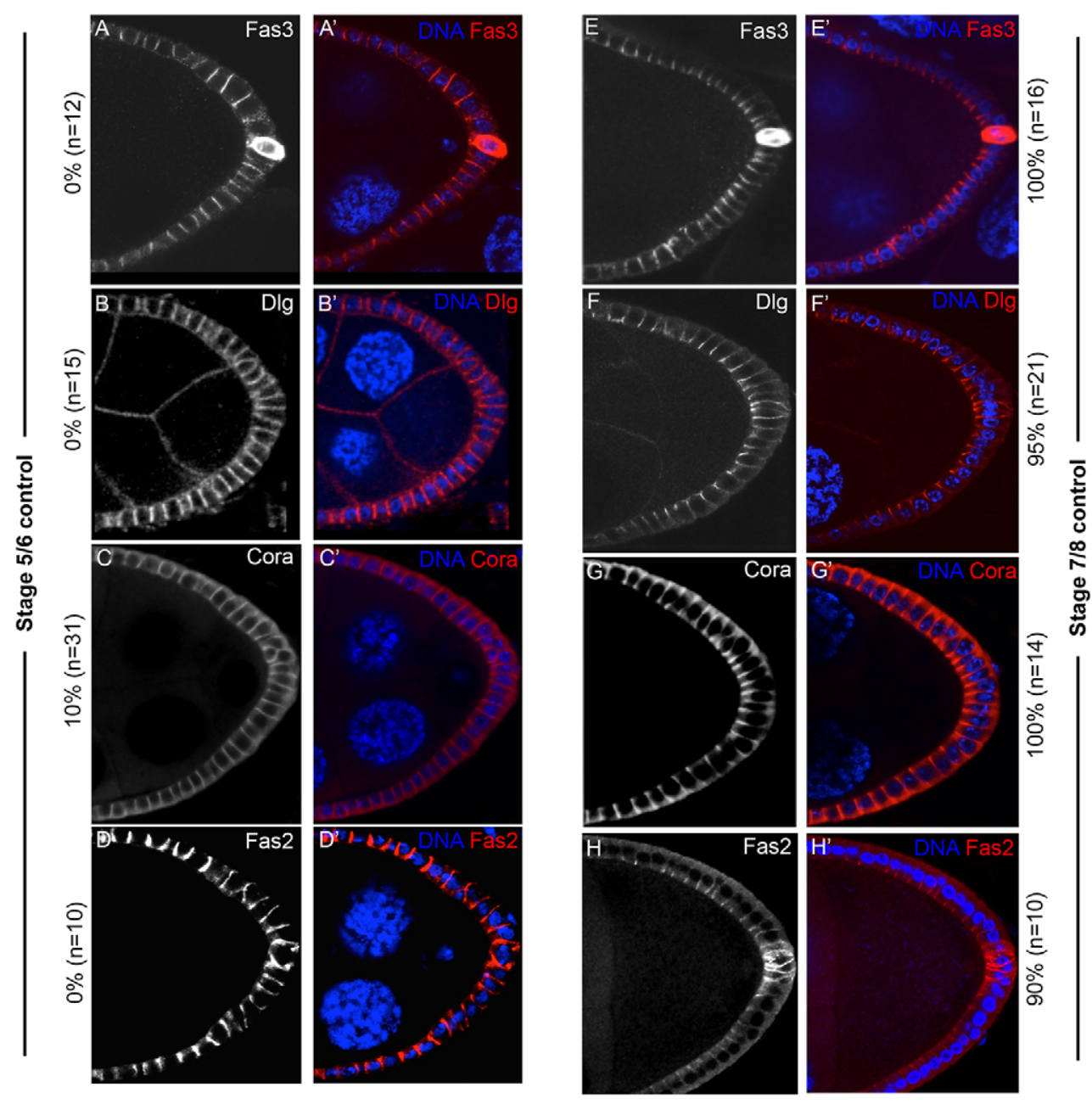

Fig. 4. Components of the septate junctions display similar localization patterns during oogenesis. (A-D') During early oogenesis, the septate junction (SJ) proteins Fas3 $\left(A, A^{\prime}\right)$, Dlg $\left(B, B^{\prime}\right)$, Coracle (Cora; $\mathrm{C}, \mathrm{C}^{\prime}$ ) and Fas2 (D, $\left.\mathrm{D}^{\prime}\right)$ are expressed uniformly along the lateral membrane. $\left(\mathrm{E}-\mathrm{H}^{\prime}\right)$ From S7, an apicolateral concentration of Fas3 $\left(E, E^{\prime}\right)$, Dlg $\left(F, F^{\prime}\right)$, Cora $\left(\mathrm{G}, \mathrm{G}^{\prime}\right)$ and Fas2 $\left(\mathrm{H}, \mathrm{H}^{\prime}\right)$ becomes distinct, especially in the posterior FCs. The percentages indicate the frequency at which these SJ proteins localize apicolaterally. All merged images show DAPI in blue, and Fas3, DIg, Fas2 and Cora in red.

(Arm) and E-Cadherin (Shotgun - FlyBase) are largely unaffected in $\alpha$-Spec FCs (Arm $87.5 \%$ as control, $n=16$; E-Cadherin $93 \%$ as control $n=14$; Fig. S2E-I; data not shown).

\section{$\alpha$-Spectrin mutant cells fail to form a columnar epithelium}

We noticed that $\alpha$-Spec FCCs appear to have a reduced apical-tobasal length (Fig. 5A). Regulation of epithelial cell shape, such as changes in relative sizes of apical, basal and lateral membranes, is key to morphogenesis. FCs undergo various morphogenetic changes, including the transformation from a cuboidal to a columnar epithelium at mid-oogenesis (Dobens and Raftery, 2000). PFCs increase their height four times from $\mathrm{S} 4 / 5$ to $\mathrm{S} 9$, but their width remains constant (Fig. S1; Fig. S3A), supporting previous conclusions that $\mathrm{FC}$ columnarization does not result from a decrease in apical surface, but rather from an expansion of the lateral membrane, probably driven by cellular growth (Kolahi et al., 2009). To test whether columnarization is aberrant in $\alpha$-Spec FCCs, we measured the height and width of S9 $\alpha$-Spec PFCs. The mean height of control S9 FCs is $18.95 \mu \mathrm{m}$, whereas that of $\alpha$-Spec S9 FCs is $12 \mu \mathrm{m}$ (Fig. $6 ; n=40$ ), suggesting that $\alpha$-Spec mutant cells fail to become columnar. By contrast, the width of $\alpha$-Spec S9 cells is similar to control cells (Fig. 6). Thus, at mid oogenesis, $\alpha$-Spec cells maintain a cuboidal shape, whereas control cells undergo columnarization by growing longer lateral membranes.

The failure of $\alpha$-Spec FCCs to form a columnar epithelium is not a consequence of the tissue architecture being disrupted, as the above measurements were performed in mutant monolayers. Our findings show that SBMS is required for the control of cell shape not only in erythrocytes, but also in epithelial cells. Furthermore, both cell shortening and aberrant localization of SJ components are cellautonomous defects of $\alpha$-Spec FCCs within a monolayered epithelium, thus possibly preceding the onset of multilayering.

\section{Similarities between $\alpha$-Spectrin and integrin mutant follicle cells}

The phenotype of $\alpha$-Spec mutant FCs resembles loss of integrin function. Cells mutant for myospheroid (mys), which encodes for the only $\beta$-chain in the ovary (Devenport and Brown, 2004; Fernández-Miñán et al., 2007), form multilayers, and display differentiation and polarity defects at the ectopic layers, but exit mitosis properly. In addition, we have previously shown that integrins regulate cell shape in the wing epithelium (DominguezGimenez et al., 2007). Similarly, mys FCs also show shape defects, with a reduced height, but a similar width, compared with control FCs (Fig. S3B). Thus, both integrins and $\alpha$-Spec are required for apical contraction-independent cell elongation during FE morphogenesis.

We noticed that $\alpha$-Spec mutant FCs accumulate more F-actin (Fig. 6C, inset; Fig. S4A,B). The actomyosin cytoskeleton has been repeatedly linked to cell shape changes. Rhol and its effector Myosin II regulate apical-basal length of wing disc cells (Widmann and Dahmann, 2009), and FCs mutant for Rok (Rho kinase) or for the regulatory chain of Myosin II [also known as MRLC or spaghetti squash $(s q h)$ ] present an abnormal shape (Wang and 

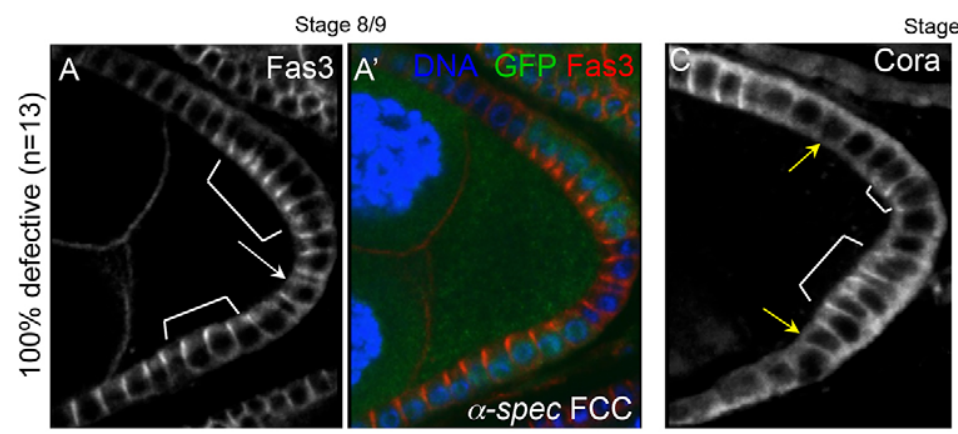

age $8 / 9$
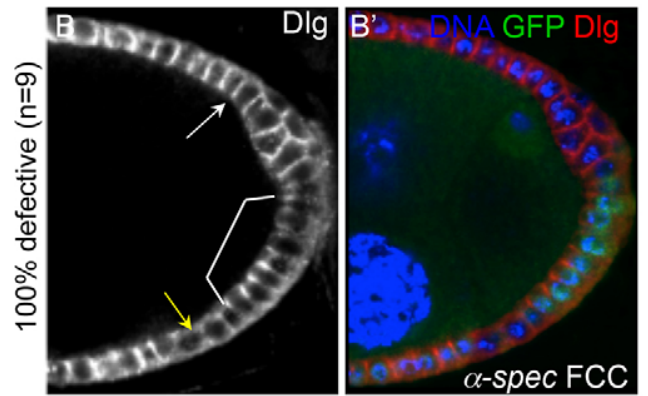
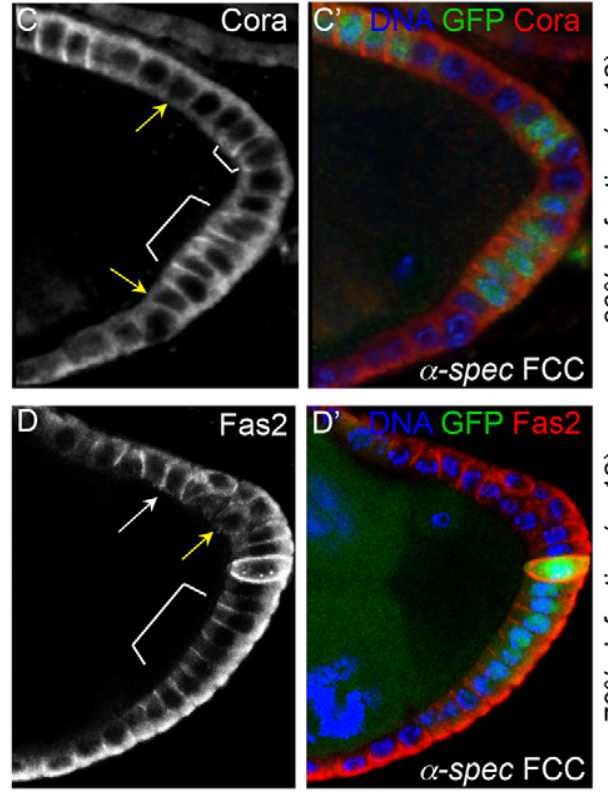

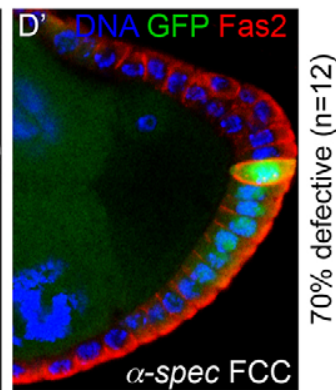

Fig. 5. The apicolateral localization of Fasciclin 3, Discs large, Coracle and Fasciclin 2 is lost in $\alpha$-Spectrin mutant cells. (A-D') At S8/9, the apicolateral localization of the SJ proteins is lost in $\alpha$-Spec mutant FCs ( $\alpha$-Spec FCCs) that form a monolayered FE (arrows), compared with their neighboring control FCs (brackets). The proteins sometimes extend along the entire lateral membrane (white arrows) or are absent altogether (yellow arrows). The percentages indicate the ratio of egg chambers containing partial posterior clones in a monolayer that display this defect. The number of mutant cells in each egg chamber was at least five. All merged images show DAPI in blue, and Fas3, Dlg, Cora or Fas2 in red. $\alpha$-Spec FCCs lack GFP.
Riechmann, 2007). The spectrin cytoskeleton provides erythrocytes with mechanical properties, and is somehow functionally linked to the actomyosin network in the Drosophila eye (Deng et al., 2015). Clear functional interactions also exist between integrins and actomyosin (He et al., 2010), and we have shown here that $\alpha$-Spec and mys FCs show similar phenotypes, including aberrant shape. These observations prompted us to test whether integrins and $\alpha$-Spec interact with the Rho1-myosin pathway during FE morphogenesis.

\section{Higher Rho1 or Sqh activity, and lower Myosin II activity, increases and decreases $\alpha$-Spectrin epithelial integrity defects, respectively}

To study whether $\alpha$-Spec might interact with the actomyosin cytoskeleton in regulating FE architecture, we first analyzed the effects of increasing myosin activity in cells that lack $\alpha$-Spec, using several strategies. Firstly, we overexpressed Sqh (fused to GFP or mCherry) in $\alpha$-Spec FCC-containing egg chambers, and found it to increase S3-6 multilayers from $48 \%(n=25)$ to $66 \%(n=27)$ (Fig. 7).
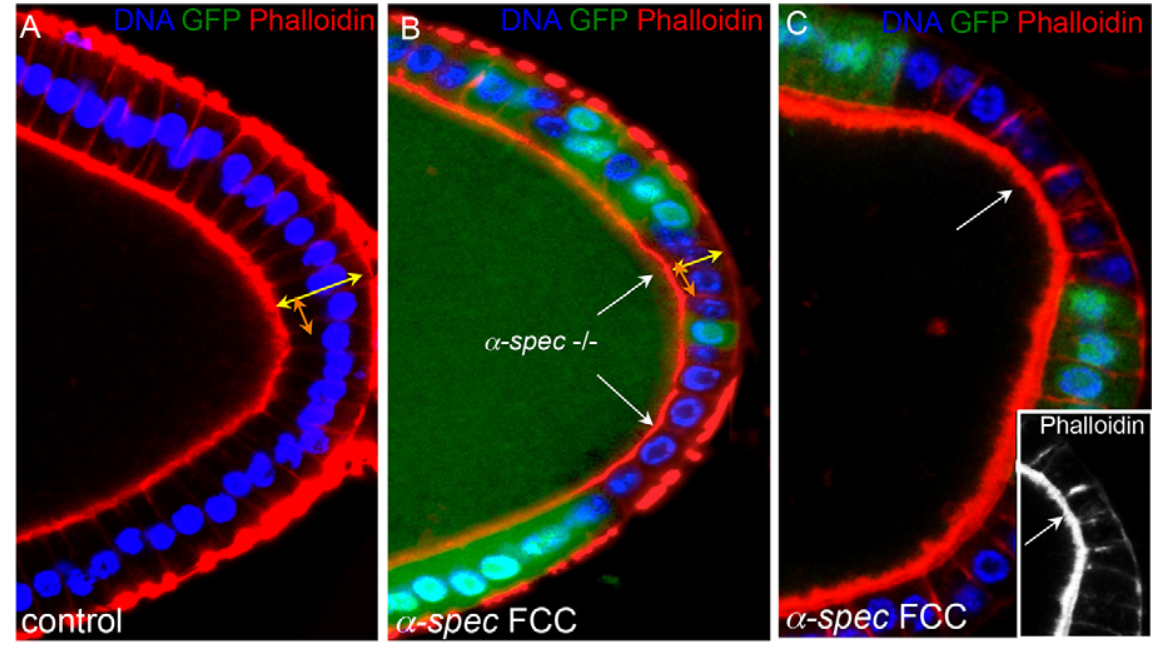

D $25 . \quad$ Cell height (S9)

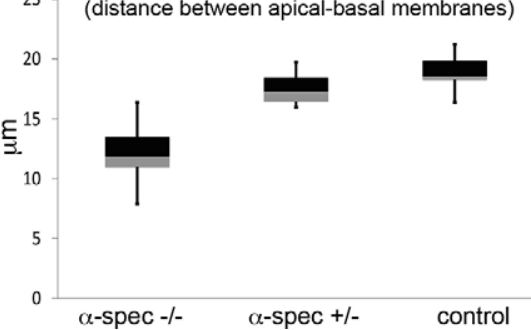

Cell width (S9)

(distance between lateral membranes)

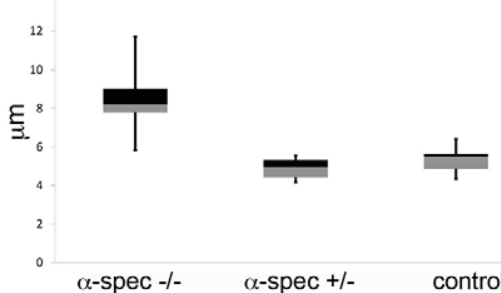

Fig. 6. $\alpha$-Spectrin mutant follicle cells fail to form a columnar epithelium. (A) Most FCs expand their lateral membrane at mid-oogenesis, changing shape from cuboidal to columnar (see also Figs S1 and S4). (B,C) Contrary to wild-type FCs, $\alpha$-Spec cells ( $\alpha$-Spec FCCs, $\alpha-\mathrm{Spec}^{-1-}$ ) do not extend properly their lateral membrane and fail to become columnar. (C) F-actin levels appear to be higher in $\alpha$-Spec mutant cells. A-C show posterior FCs. DAPI in blue, Phalloidin in red. All $\alpha$-Spec cells lack GFP. White arrows indicate the mutant clone. (D) Box plot quantification at S9. The mean height (yellow arrows in $A, B$ ) of wild-type and $\alpha$-Spec cells is 18.95 and $12 \mu \mathrm{m}$, respectively. The mean width (orange arrows in $A, B$ ) of wild-type and $\alpha$-Spec cells is 5.30 and $8.44 \mu \mathrm{m}$, respectively. The Welch two-tailed $t$-test $P$-values between wild type and $\alpha$-Spec mutant $(-/-)$ for cell height and width are $1.123 \times 10^{-06}$ and 0.0002 , respectively. Measurements were performed in posterior cells that maintain a monolayer. $n=40$ (four cells in ten egg chambers) for all samples. 

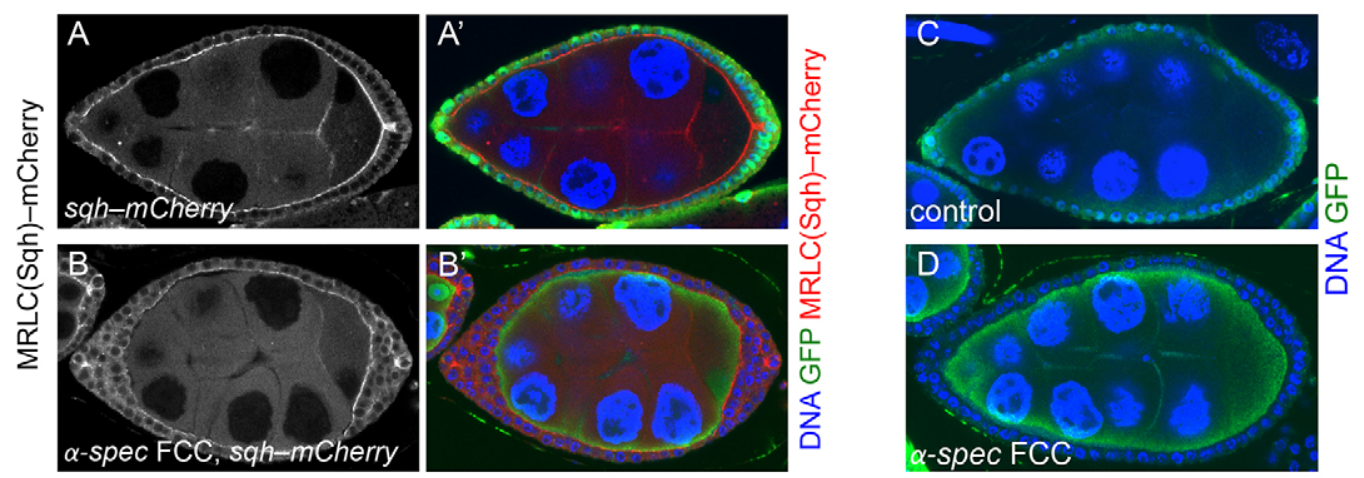

E

$\square$ Monolayered $\square$ Bilayered $\square$ Multilayered

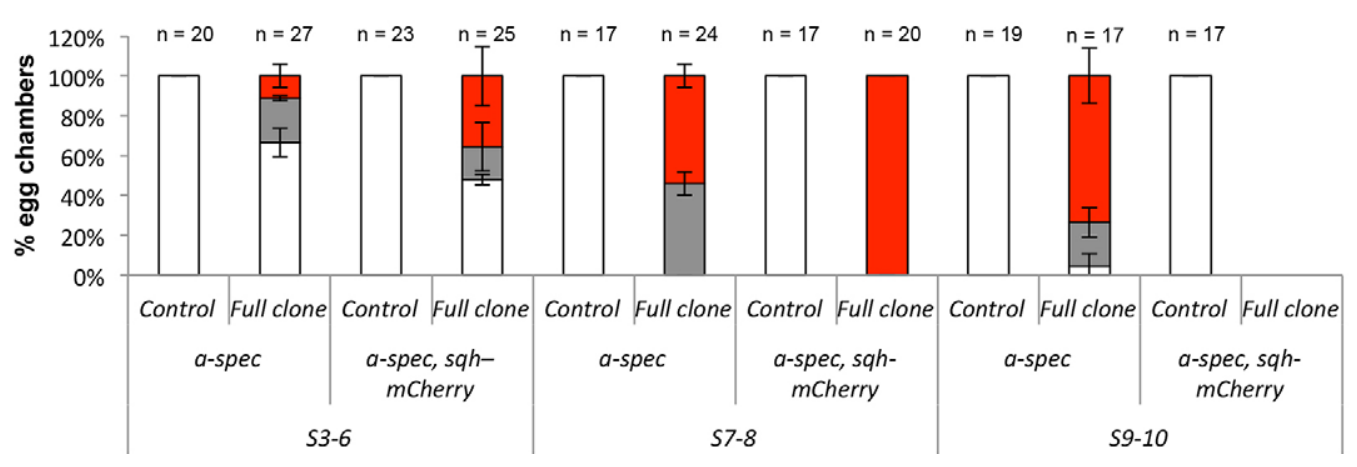

Fig. 7. Overexpressing the Myosin Regulatory Light Chain spaghetti squash in egg chambers with $\alpha$-Spectrin mutant clones worsens the multilayering phenotype. (A-D) Sqh-mCherry S8/9 egg chambers without $\alpha$-Spec mutant FCs (A, $\left.\mathrm{A}^{\prime}\right)$, or with large $\alpha$-Spec clones $\left(\alpha-S p e c\right.$ FCCs, B, $\left.\mathrm{B}^{\prime}\right)$ are compared with egg chambers which contain large $\alpha$-Spec clones only (D). Control egg chambers contain no $\alpha$-Spec clones (A,A',C). Mutant clones lack GFP.

$\left(A-B^{\prime}\right)$ Sqh-mCherry is in red (right panels) or white (left panels). (E) Quantification of the multilayering phenotype reveals a worse defect in egg chambers with large $\alpha$-Spec clones and one extra copy of $s q$ (compare Full clone $\alpha$-Spec,sqh-mCherry with Full clone $\alpha$-Spec). 'Multilayered' refers to more than two layers. To simplify the analysis, we only quantified full or very large mutant clones. Cells that express Sqh-mCherry and are heterozygous for $\alpha$-Spec do not show bilayers (control $\alpha$-Spec,sqh-mCherry and A, $\left.\mathrm{A}^{\prime}\right)$. We did not obtain any S9-10 $\alpha$-Spec egg chambers that also overexpress Sqh. Error bars indicate two different experiments.

This multilayering enhancement is more dramatic at mid-oogenesis, as all $\mathrm{S} 7 / 8$ egg chambers show more than two layers when an extra copy of Sqh was expressed in $\alpha$-Spec cells $(n=20)$, compared with $50 \%$ when the FCs were only mutant for $\alpha$-Spec $(n=24)$ (Fig. 7E; Fig. S5A-C). The fact that all $\alpha-S p e c$ S7/8 egg chambers display ectopic layers when Sqh was overexpressed prevented us from assessing whether increased myosin enhanced the cell shape phenotype.

Secondly, we increased Rho1 levels in $\alpha$-Spec mutant cells. We were unable to obtain an FE that overexpressed Rho1 (GFP- or mCherry-tagged) and also had $\alpha$-Spec FCCs. Occasionally, we obtained a few cells of the right genotype, but they showed clear signs of undergoing death (data not shown). As an alternative, we overexpressed Rho1 in $\alpha$-Spec mutant cells by driving expression of $U A S-\alpha$-SpecRNAi, UAS-Rhol or UAS-constitutively active-(CA)Rhol by the FC-specific driver trafficjam(tj)-Gal4. S7/8 egg chambers expressing $\alpha$-SpecRNAi in FCs show multilayers in $33 \%$ of the cases $(n=12)$, whereas overexpression of Rhol or $C A$ Rhol in these cells increases the multilayering phenotype to $50 \%$ $(n=12)$ and $80 \%(n=10)$, respectively.

We then tested whether a reduction in myosin activity might rescue the $\alpha$-Spec multilayering. To do this, we reduced the levels of the Myosin II gene zipper (zip) by driving the expression of UASzipRNAi with tj-Gal4, and inducing $\alpha$-Spec FCCs in the same epithelium. Similarly, we expressed a zip-dominant negative-(DN) form in cells that are also mutant for $\alpha$-Spec (Fig. S6). In both cases, we observed a reduction in the $\mathrm{S} 7 / 8$ multilayering phenotype, from $90 \%$ (control, $n=10)$ to $45 \%$ (zipRNAi, $n=11$ ), and from $50 \%$ (control, $n=10$ ) to $20 \%$ (zipDN, $n=10$ ).

As elimination of integrins affect FCs similarly to the loss of $\alpha$ Spec, we next tested whether integrins might also interact with the actomyosin cytoskeleton to control FE architecture. Indeed, we find that overexpression of Sqh (fused to either GFP or mCherry) in egg chambers containing mys mutant FCs increases the percentage of S3-6 follicles with multilayers from $42 \%$ (mys alone, $n=21$ ) to $68 \%$ (sqh-GFP, $n=97$ ) or $82 \%$ (sqh-mCherry, $n=56)$ (Fig. 8).

To investigate how specific this increased stratification effect of myosin is, we analyzed the impact that overexpressing Sqh has on the multilayering phenotype of bazook ${ }^{815} \mathrm{FCs}$. We observe that increasing myosin activity does not enhance bazook $a^{815}$ multilayers (Fig. S5D-F). All these results together suggest that spectrins and integrins are required to maintain the proper levels of actomyosin activity in the monolayered FE. This is further supported by the fact that both F-actin and Sqh are misplaced and expressed at higher levels in $\alpha$-Spec mutant cells (Fig. 6C; Fig. S4).

\section{$\alpha-$ Spectrin is required for egg chamber morphogenesis and egg size}

Defects in FE tension and morphogenesis are likely to result in egg chambers with aberrant shapes (Wang and Riechmann, 2007). Young egg chambers are spherical, elongating from S5 along the anteriorposterior (AP) axis to create the elliptical shape of the egg. We noticed 

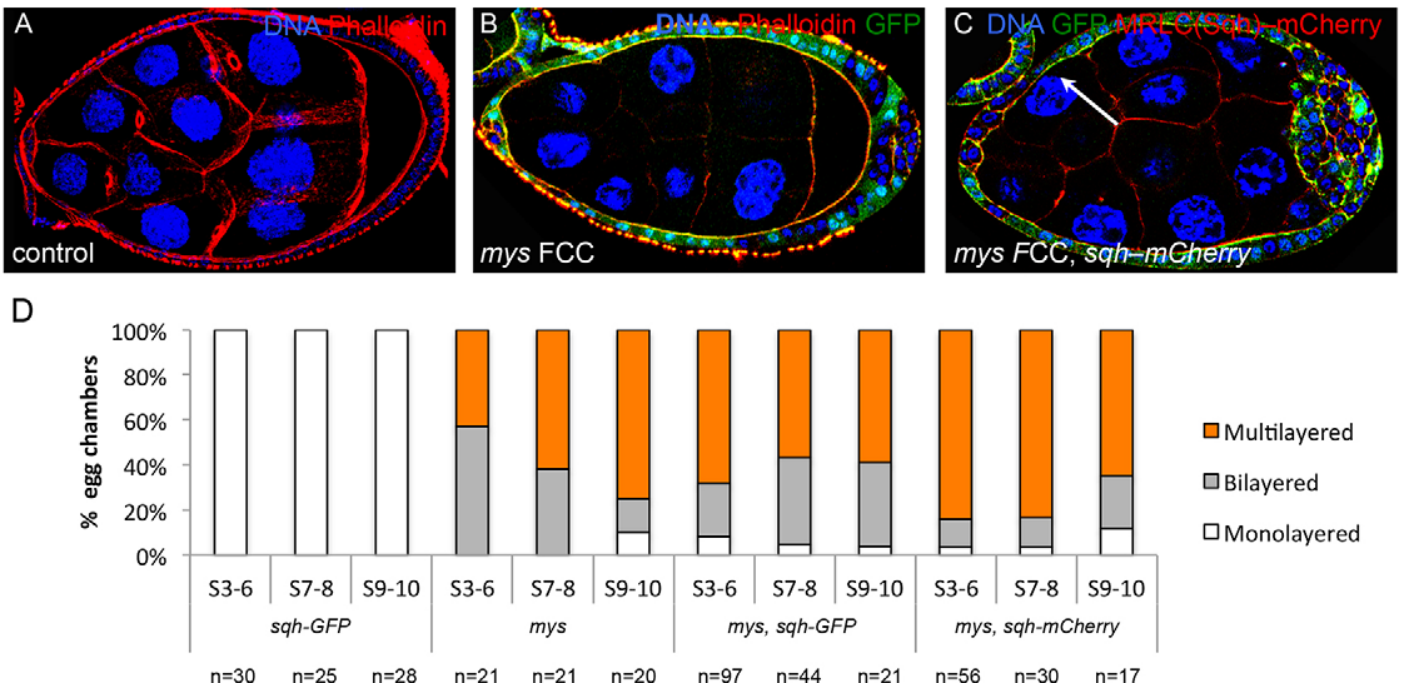

Fig. 8. Over-expressing the Myosin Regulatory Light Chain spaghetti squash in mys cells enhances the multilayering phenotype. (A-C) S8/9 egg chambers stained for TOPRO (blue, A-C), GFP (green, B,C) and F-actin (A,B, red) or Sqh-mCherry (C, red). Mutant clones lack GFP. (A) Wild type. (B) mys mutant clones (FCCs) develop multilayers at the posterior pole. (C) This phenotype is enhanced when an extra copy of sqh (sqh-mCherry) is expressed in these egg chambers. (D) Quantification of the multilayering phenotype in mys, sqh-GFP, mys,sqh-GFP and mys,sqh-mCherry egg chambers.

that $\alpha$-Spec egg chambers seemed rounder than wild-type egg chambers (Figs 1, 3, 7). In fact, S8/9 mutant egg chambers with a multilayered $\mathrm{FE}$ either at the anterior, or both anterior and posterior poles, had a shorter AP axis (Fig. S7). This does not seem to be the case when multilayers were only present at the posterior, as the AP axis was not statistically different from controls. Even though mutant egg chambers seem rounder, the dorsoventral axis is slightly shorter in $\alpha$-Spec egg chambers than in controls, suggesting that the total volume of the germline in $\alpha$-Spec egg chambers is smaller.

Egg chambers with integrin mutant FCs render rounder eggs (Bateman et al., 2001). To study whether this is similar in eggs resulting from $\alpha$-Spec mutants, we measured the AP axis in eggs obtained from $\alpha$-SpecRNAi, tj-Gal4 egg chambers, and observed that, similar to integrins, these eggs also show a reduced AP axis compared with controls (Fig. S7C).

\section{DISCUSSION}

Revised roles for the spectrin cytoskeleton in regulating proliferation, differentiation and polarity

We find that in the germline $\alpha$-Spec is not a major regulator of the Hippo pathway. Mutations in hippo, $\beta$-Spec or $\alpha$-Spec result in a stratified FE, but contrary to previous interpretations, and unlike Hippo, spectrins are not required for the FCs to exit mitosis. We believe that the suggestion that Spec mutant FCs over-proliferate was an over-interpretation from the multilayering phenotype, as $\alpha$ Spec cells were not checked for mitotic markers in that report (Fletcher et al., 2015). Again unlike hippo, $\alpha$-Spec mutant PFCs only show defects in differentiation when they are located in the ectopic layers of the stratified FE, and oocyte polarity is largely unaffected in mutant egg chambers. It was recently shown that a $\beta$-Spec allele with a premature stop codon at amino-acid 1046 partially phenocopies hippo, with strong defects in FE integrity, actin organization and oocyte polarity (Wong et al., 2015). The null $\beta$-Spec ${ }^{\mathrm{G} 113}$ mutant allele (Hulsmeier et al., 2007) behaves similarly to $\alpha$-Spec mutants, showing Hnt defects mainly in ectopic layers (Fig. S8A-B,F), but Fas3 mislocalization in monolayers (Fig. S8C-D'). More importantly, $\beta-$ Spec $^{\mathrm{G} 113}$ FCCs exit mitosis properly (Fig. S8A, $\mathrm{A}^{\prime \prime \prime}$ ). The differences observed between the two $\beta$-Spec alleles are likely to be due to the fact that $\beta-\operatorname{Spec}^{\mathrm{G} 113}$ is a null allele.
In conclusion, $\alpha$-Spec and $\beta$-Spec FCCs do not phenocopy hippo mutants when the cells are part of a monolayer, and they seem to adopt a partial hippo-like differentiation phenotype only when positioned at ectopic layers, even though $\alpha$-Spec and $\beta$-Spec cells never divide after S6. Thus, the main function of the spectrin cytoskeleton in FCs is not proliferation control or regulation of the Hippo pathway, although an interaction between spectrins and Hippo might occur once the FCs are within an aberrantly organized FE. The function of spectrins in FCs is in contrast with other tissues, where $\alpha$ - and $\beta$-Spec appear to regulate growth through Hippo (Deng et al., 2015; Fletcher et al., 2015; Wong et al., 2015).

\section{$\alpha$-Spectrin and $\beta$-Spectrin, but not $\beta_{H^{-}}$Spectrin, mutant epithelia fail to maintain a monolayered architecture}

Similar to Hippo, $\alpha$-Spec and $\beta$-Spec are required for the FE to maintain a monolayer. There is an increase in the multilayering phenotype in egg chambers with large clones from $\mathrm{S} 3 / 6$ to $\mathrm{S} 7 / 8$ [36\% $(n=37)$ and $100 \%(n=34)$, respectively; Fig. S9A-B]. Also, the presence of control cells in $\alpha$-Spec mosaic epithelia aids the mutant cells to maintain a monolayer from $\mathrm{S6}$, as there is a higher percentage of S7-9 egg chambers with multilayers when the FE contains large $\alpha$-Spec clones (Fig. S9C, 'full'; 95\%, $n=54$ ) than when the mutant clone is only at the posterior end (Fig. S9C, 'post'; $53 \%, n=49$ ). The control of FE architecture appears to be mediated by the lateral spectrin network. Loss of $\alpha$-Spec seems to disrupt both lateral $(\alpha / \beta)$ and apical $\left(\alpha / \beta_{\mathrm{H}}\right)$ SBMS in the FE, as $\beta$ and $\beta_{\mathrm{H}}$ subunits are no longer localized laterally and apically in $\alpha$-Spec cells (Fig. S8G; Lee et al., 1997), but no multilayering was reported for $\beta_{H}$-Spec egg chambers, in which a loss of apical $\alpha$-Spec was observed (Zarnescu and Thomas, 1999), suggesting that the loss of the lateral $\alpha / \beta$ is responsible for the FE stratification. Also, $\beta_{\mathrm{H}^{-}} \mathrm{Spec}$ is mislocalized in sosie mutants, but the $\mathrm{FE}$ architecture is maintained (Urwyler et al., 2012).

\section{A novel function for $\alpha$-Spectrin in localizing septate junction components}

Incipient SJs are first detected between the FCs with the completion of proliferation at S6 (Mahowald, 1972; Müller, 2000; this work). We show here that the localization of several SJ components is 
affected in $\alpha$-Spec FCCs, suggesting that spectrins are required for proper SJ formation. This is further supported by other observations. First, Fas 3 localization is affected in $\beta$-Spec FCCs. Second, Neuroglian (an SJ component) is required for maintaining the stability of the FE (Wei et al., 2004). Third, the reduction of both $\alpha$ and $\beta$-Spec leads to mislocalization of Dlg, Neuroglian and Fas 2 in neuromuscular junctions (Featherstone et al., 2001; Pielage et al., 2005). And fourth, it has been suggested that the SBMS and ankyrin associate with SJ components (Bennett and Chen, 2001; Dubreuil et al., 2001).

As the mislocalization of SJ components in Spec mutant FCCs is observed in monolayers, and thus prior to the onset of stratification, we speculate that Spec-dependent distribution of SJ components might contribute to the Spec function in the epithelium. This idea is supported by Crumbs overexpression, which leads to defects in SJs and ZA, and multilayering of the ectoderm cells (Klebes and Knust, 2000; Wodarz et al., 1995), and by dpak (Pak - FlyBase) FCs, which mislocalize Fas3 and show multilayering and columnarization defects (Conder et al., 2007). Furthermore, the aberrant accumulation of Fas 2 at the lateral membrane of Tao FCs prevented membrane shrinking in the cuboidal-to-squamous transition (Gomez et al., 2012). However, fas3, fas 2 and cora mutant cells do not show shape defects or multilayering (data not shown). Thus, if SJ components contribute to the $\alpha$-Spec phenotype at all, it might be not because they are absent in $\alpha$-Spec mutant cells, but because they are not properly distributed.

\section{A novel function for $\alpha$-Spectrin in apical constriction- independent cell elongation}

Transitions between squamous, cuboidal and columnar epithelial cell shapes are common during development, and contribute to the morphogenesis of tissues. Here, we demonstrate a cell-autonomous role for $\alpha$-Spec in promoting the cuboidal-to-columnar shape transition of the FCs. It is important to point out that the FE undergoes lateral elongation without apical constriction (Kolahi et al., 2009; Fig. S3), which might allow phenotypes to be interpreted in a simpler manner. This morphogenetic FC behavior is similar to that of vertebrate neuroepithelia, where cell elongation precedes apical constriction (Suzuki et al., 2012), and it would be interesting to study the function of Spec in the columnarization of these cells.

Although the molecular mechanism of apical constrictionindependent cell elongation is unknown, we think that a primary role for the SBMS lies in facilitating changes in cell shape, which is further supported by the cell shape defects in $\alpha$-Spec gut epithelia (Lee et al., 1993), perhaps by contributing to the proper distribution of adhesion molecules. This function of the SBMS in membrane biology is conserved in other cells, as spectrins stabilize the plasma membrane during blastoderm cellularization (Pesacreta et al., 1989), and control photoreceptor morphogenesis through the modulation of membrane domains (Chen et al., 2009; Williams et al., 2004). The spectrin cytoskeleton might also impact on FE columnarization by interacting with the actomyosin cytoskeleton. It is known that apical-basal elongation in drebrin E (drebrin 1) depleted human Caco2 cells is impaired, as a possible consequence of the lack of interaction between drebrin $\mathrm{E}$ with spectrins and actomyosin (Bazellieres et al., 2012). Also, the elongation of neuroepithelial cells depends on the assembly of an actomyosin network in the apical junctional complex, regardless of whether cells are constricting or not (Hildebrand, 2005). In Drosophila wing discs, the Rho1-Myosin II pathway at the apicolateral membrane seem to regulate the cuboidal-to-columnar shape transition, whereas in the germline, Rok and sqh mutant FCs fail to adopt a normal shape
(Wang and Riechmann, 2007). Finally, SBMS seems to modulate cortical actomyosin contractility in the eye (Deng et al., 2015), and possibly in the FE (this work; Wong et al., 2015). Together, these data suggest that Myosin II activity is aberrant in $\alpha$-Spec mutant $\mathrm{FCs}$, contributing to defects in columnarization and FE architecture.

\section{Integrins, spectrins and the actomyosin cytoskeleton}

Increasing Rhol and Sqh activities enhances the Spec multilayering phenotype, whereas reducing Myosin II activity decreases it. In addition to this functional link between the SBMS and the RhoMyosin pathway, we also show that mys cells fail to columnarize, and that an extra copy of $s q h$ increases the mys multilayering phenotype. It has been shown that integrins regulate the RhoMyosin pathway to induce actomyosin-generated forces (Geiger et al., 2009). Thus, as is the case for spectrins, integrins might also control cell shape and epithelia morphogenesis by modulating the actomyosin activity.

How the SBMS and integrins might modulate actomyosin is unknown, and one possible mechanism is by regulating Myosin II activity directly. However, we would like to propose an alternative mechanism. Spectrins can bind F-actin, and integrins and spectrins interact with proteins involved in the association of $\mathrm{F}$-actin with the membrane (Beaty et al., 2014; Médina et al., 2002). Furthermore, $\alpha$-Spec and integrins regulate the actin cytoskeleton through Rac (Bialkowska et al., 2005). Previous studies have shown that both $\beta$-Spec and mys mutant FCs display similar defects in the basal level of F-actin (Delon and Brown, 2009; Wong et al., 2015), which are recapitulated in $\alpha$-Spec mutant cells (Fig. S4A). Thus, any defects in actin organization in mys and Spec mutant FCs could in turn result in defects in the activity of Myosin II.

Regardless of whether integrins and spectrins regulate F-actin or myosin, or both, spectrins and integrins might act together. The SH3 domain of $\alpha$-Spec interacts with Tes (Rotter et al., 2005), a component of integrin-dependent focal adhesions (Coutts et al., 2003), and mammalian $\alpha$ II-Spec stabilizes $\beta 3$-integrin anchorage, suggesting $\alpha$ Spec as a physical link between focal adhesions and F-actin (Ponceau et al., 2015). In the FE, we observe that $\alpha$-Spec and $\alpha$ PS1 colocalize in the lateral, and possibly apical, membrane (Fig. S10). In addition, we show that the localization of $\alpha-S p e c$ in mys clones, and the localization of $\beta \mathrm{PS}$ in $\alpha$-Spec mutant clones, is majorly unaffected (Figs S11, S12). Furthermore, we find that expression of a constitutively active integrin that reduces multilayering of mys FCCs (Fernández-Miñán et al., 2007; Meignin et al., 2007), fails to rescue $\alpha$-Spec multilayers (data not shown). Thus, we would like to propose that $\alpha$-Spec and integrins act independently of each other, but as part of the same functional complex regulating the actomyosin cytoskeleton and tissue architecture.

\section{What is important for maintaining a monolayered epithelium?}

An early event following oncogenic mutations in an epithelium is the escape of the daughter cells from the monolayered epithelium, forming disorganized masses. Spindle orientation has been linked to tumor-like growth in various tissues, and we find that there is a good correlation between spindle misorientation and 'tumor-like masses' at the FE: hippo, mys and $\alpha$-Spec FCCs show misaligned spindles and severe multilayering (Fig. S13; Fernández-Miñán et al., 2007; Meignin et al., 2007), whereas Notch FCCs, which overproliferate, do not show multilayering or spindle orientation defects (data not shown). However, perpendicular divisions alone are insufficient to promote stratification, and a mechanism, depending on lateral cellcell adhesions, is in place to avoid multilayering as a sole 
consequence of spindle misorientation (Bergstralh et al., 2013, 2015). We would like to propose that spindle misorientation contributes to FE disorganization, but that this 'safeguard' mechanism is somehow inactive in hippo, mys and Spec mutant FCCs. What other aspect of the mutant phenotypes might then be linked to multilayering? A clue might come from the Spec mutant and mys FCCs. First, there is an increase in the $\alpha$-Spec multilayers after S6, when both FCs and egg chambers undergo various morphogenetic changes. Second, the volume of the germline surrounded by large $\alpha$-Spec FCCs appears smaller. And third, Myosin II activity is increased in $\alpha$-Spec and mys mutant cells. In our interpretation of the results, a proper distribution of Myosin II activity in a Spec- and integrin-dependent manner allows the right amount of forces to be distributed across the membrane and the epithelium. Thus, it is possible that proper cell-cell interactions, adequate force balance and precise spindle orientation are key to maintaining a monolayered epithelium, especially upon the mechanical stress induced by morphogenesis.

\section{MATERIALS AND METHODS}

\section{Fly stocks and creation of follicle cell clones}

FRT2A $\alpha$-Spec ${ }^{r g 41}: \alpha-$ Spec $^{r g 41}$ is an allele containing a 20-base pair deletion that creates a frame-shift and premature termination near the $5^{\prime}$-end of the transcript, and is a null allele (Fig. S1C-D). The original study by Lee et al. (1997) created excision clones of a $p[>l a c Z, \alpha-s p e c>]$ construct in flies transheterozygous for the $l(3) \alpha-$ spec $^{r g 41}$ chromosome and the deficiency $D f(3 L) R-R 2$. This deficiency eliminates $\alpha$-Spec, but also $d l t$, which encodes a protein required for FE formation and polarity (Bhat et al., 1999; Klebes and Knust, 2000). Because of this, heterozygosis of $d l t$ in the cells that lack the $p[>$ lac $Z, \alpha$-spec $>$ ] construct might have influenced the described phenotypes, and these phenotypes might not be only due to the lack of $\alpha$-Spec.

$s q h-G F P$ and $s q h-m C h e r r y$ are fusion proteins expression of which is driven by the endogenous $s q h$ regulatory sequences and can substitute the sqh gene (Martin et al., 2009; Royou et al., 2004). Stocks used were: FRT42Dhippo $^{42-47}$ (Wu et al., 2003), FRT19AB-spec G113 (Hulsmeier et al., 2007), FRT101mys ${ }^{11}$ (also known as mys ${ }^{X 43}$ ) (Bunch et al., 1992), FRT19Abazooka ${ }^{815}$ (Djiane et al., 2005), FRT40Fas ${ }^{\text {A142 }}$ (Wells et al., 2013), FRT19AFas2 ${ }^{\text {G0336 }}$ (Szafranski and Goode, 2007), FRT42Dcoral (Laprise et al., 2009), UAS-Rhol (Bloomington-7334), UAS-constitutively active-CA-Rhol (Bloomington-8144), UAS-zip ${ }^{R A i}$ (VDRC7819), UASzipDN (Monier et al., 2010), UAS- $\alpha$-spec ${ }^{R N A i}$ (VDRC25387).

To generate most follicle cell mutant clones, we used the heat shock flipase (hs-flp) system (Chou and Perrimon, 1992). Mutant clones were marked by the absence of GFP or RFP (Xu and Rubin, 1993). The heat shock was performed at $37^{\circ} \mathrm{C}$ for $2 \mathrm{~h}$ over 3 days during third instar larval. To generate mys and baz follicle cell clones, we used the FRT/FLP technique combined with the Gal4 system. The e22c-Gal4 driver is expressed in the follicle stem cells in the germarium and was used in combination with UAS-flp.

The Sqh experiments in Figs 7 and 8 and Fig. S5 used flies that contain three copies of the $s q h$ gene: two endogenous ones and the fluorescently tagged version, which is expressed under the sqh promotor.

All UAS constructs were expressed by the FC-specific driver traffic-jamGal4 (DGRC104055), except for the UAS-zipDN experiment, which was expressed by the MARCM system (mosaic analysis with a repressible cell marker). In this case, hs-flp expression was induced by heat-shocking only adults, at $37^{\circ} \mathrm{C}$ for $1 \mathrm{~h}$, over 2 days. GFP-positive cells were either $\alpha$-Spec mutant or $\alpha$-Spec mutant overexpressing zipDN-GFP.

All females analyzed were between 3 and 10 days old.

Full names of genotypes shown in each figure are listed in supplementary Materials and Methods.

\section{Antibodies}

Primary antibodies were: rabbit anti- $\beta-$ Spec (1:500; gift of Dr Klämbt, University of Münster, Germany), mouse anti-Fas2 [1:100; 1D4, Developmental Studies Hybridoma Bank (DSHB)], chicken anti-GFP
(1:2000; ab13970, Abcam), mouse anti-Arm (1:200; N2 7A1, DSHB), rat anti-E-Cadherin (1:200; DCAD2, DSHB), mouse anti- $\alpha$-Spec (1:250; 3A9, DSHB), rat anti-Tubulin (1:500; MAB 1864, Chemicon), rabbit anti-aPKC (1:1000; C-20, Santa Cruz Biotechnology), mouse anti-Dlg (1:500; 4F3, DSHB), mouse anti-Fas3 (1:100; 7G10, DSHB), mouse anti-Hnt (1:15; 1g9-s, DSHB), rabbit anti-PH3 (1:500; 06-570, Upstate Biotechnology), rabbit anti-Staufen (1:3000; gift of Dr St Johnston, Gurdon Institute, University of Cambridge, UK), mouse anti-integrin $\beta$ PS (1:50; CF.6G11, DSHB), mouse anti-Cora (1:100; gift of Dr Gardiol, Gurdon Institute, University of Cambridge, UK). Species-appropriate AlexaFluor488-, AlexaFluor568- and AlexaFluor647-conjugated secondary antibodies were used (1:100; Molecular Probes).

\section{Immunohistochemistry}

For immunostaining, we followed standard procedures (Williams et al., 2014). For F-actin staining, Alexa-coupled Phalloidin (1:200; Invitrogen) was added in $\mathrm{PBS} / 2 \%$ Tween- 20 for 30 min prior to final washes and mounting. The samples were mounted in Vectashield (Vector)+DAPI, or incubated with the DNA dye TOPRO-3 (1:1000; Molecular Probes) for $10 \mathrm{~min}$ and then mounted in Vectashield (Vector).

\section{Acknowledgements}

We thank Dr Contreras-Sepulveda for the initial analysis of $\alpha$-Spec mutants; Dr Gonzalez-Reyes for discussions and comments on the manuscript; Drs Gardiol, Sanson, Ropër, Klämbt, Thompson and St Johnston for reagents.

\section{Competing interests}

The authors declare no competing or financial interests.

\section{Author contributions}

B.F.N., G.K.S., C.S.-C.M., I.G., I.A.-G., M.D.M.-B. and I.M.P. performed experiments and data analysis. M.D.M.-B. and I.M.P. developed the concepts. G.K.S., M.D.M.-B. and I.M.P. prepared or edited the manuscript prior to submission.

\section{Funding}

This work was supported by the Singapore Ministry of Education [Master Scholarship EDUN N23-03-044 to B.F.N.]; the Biotechnology and Biological Sciences Research Council (BBSRC) [RG42522 to I.A.G.; BB/L001748/1 to G.K.S. and I.M.P.]; the Wellcome Trust [087899/Z/08/Z to I.A.G. and I.M.P.]; the Isaac Newton Trust (Cambridge, UK) [11.35(af) to I.A.-G.]; the FEDER programme [BFU2013-48988-C2-1-P to C.S.-C.M. and M.D.M.-B.]; Junta de Andalucía [Proyecto de Excelencia P09-CVI-5058 to C.S.-C.M. and M.D.M.-B.]; a Superior Council for Scientific Research (CSIC) JAE-DOC (to I.G.); and the Department of Zoology (Cambridge) and the University of Cambridge (I.M.P.). Deposited in PMC for immediate release.

\section{Supplementary information}

Supplementary information available online at

http://dev.biologists.org/lookup/suppl/doi:10.1242/dev.130070/-/DC1

\section{References}

Abdelilah-Seyfried, S., Cox, D. N. and Jan, Y. N. (2003). Bazooka is a permissive factor for the invasive behavior of discs large tumor cells in Drosophila ovarian follicular epithelia. Development 130, 1927-1935.

Bai, J. (2002). Eyes Absent, a key repressor of polar cell fate during Drosophila oogenesis. Development 129, 5377-5388.

Baines, A. J. (2003). Comprehensive analysis of all triple helical repeats in betaspectrins reveals patterns of selective evolutionary conservation. Cell. Mol. Biol. Lett. 8, 195-214.

Baines, A. J. (2009). Evolution of spectrin function in cytoskeletal and membrane networks. Biochem. Soc. Trans. 37, 796-803.

Bateman, J., Reddy, R. S., Saito, H. and Van Vactor, D. (2001). The receptor tyrosine phosphatase Dlar and integrins organize actin filaments in the Drosophila follicular epithelium. Curr. Biol. 11, 1317-1327.

Bazellieres, E., Massey-Harroche, D., Barthelemy-Requin, M., Richard, F., Arsanto, J.-P. and Le Bivic, A. (2012). Apico-basal elongation requires a drebrinE-EB3 complex in columnar human epithelial cells. J. Cell Sci. 125, 919-931.

Beaty, B. T., Wang, Y., Bravo-Cordero, J. J., Sharma, V. P., Miskolci, V. Hodgson, L. and Condeelis, J. (2014). Talin regulates moesin-NHE-1 recruitment to invadopodia and promotes mammary tumor metastasis. J. Cell Biol. 205, 737-751

Bennett, V. and Chen, L. (2001). Ankyrins and cellular targeting of diverse membrane proteins to physiological sites. Curr. Opin. Cell Biol. 13, 61-67. 
Bergstralh, D. T., Lovegrove, H. E. and St Johnston, D. (2013). Discs large links spindle orientation to apical-basal polarity in drosophila epithelia. Curr. Biol. 23, 1707-1712.

Bergstralh, D. T., Lovegrove, H. E. and St Johnston, D. (2015). Lateral adhesion drives reintegration of misplaced cells into epithelial monolayers. Nat. Cell Biol. 17 1497-1503

Bhat, M. A., Izaddoost, S., Lu, Y., Cho, K.-O., Choi, K.-W. and Bellen, H. J. (1999) Discs Lost, a novel multi-PDZ domain protein, establishes and maintains epithelial polarity. Cell 96, 833-845.

Bialkowska, K., Saido, T. C. and Fox, J. E. B. (2005). SH3 domain of spectrin participates in the activation of Rac in specialized calpain-induced integrin signaling complexes. J. Cell Sci. 118, 381-395.

Brower, D. L., Smith, R. J. and Wilcox, M. (1980). A monoclonal antibody specific for diploid epithelial cells in Drosophila. Nature 285, 403-405.

Bunch, T. A., Salatino, R., Engelsgjerd, M. C., Mukai, L., West, R. F. and Brower, D. L. (1992). Characterization of mutant alleles of myospheroid, the gene encoding the $\beta$ subunit of the Drosophila PS integrins. Genetics 132, 519-528.

Byers, T. J., Husain-Chishti, A., Dubreuil, R. R., Branton, D. and Goldstein, L. S. (1989). Sequence similarity of the amino-terminal domain of Drosophila beta spectrin to alpha actinin and dystrophin. J. Cell Biol. 109, 1633-1641.

Chen, T. W., Chen, G., Funkhouser, L. J. and Nam, S.-C. (2009). Membrane domain modulation by Spectrins in Drosophila photoreceptor morphogenesis. Genesis 47, 744-750.

Chou, T. B. and Perrimon, N. (1992). Use of a yeast site-specific recombinase to produce female germline chimeras in Drosophila. Genetics 131, 643-653.

Conder, R., Yu, H., Zahedi, B. and Harden, N. (2007). The serine/threonine kinase $\mathrm{dPak}$ is required for polarized assembly of F-actin bundles and apical-basal polarity in the Drosophila follicular epithelium. Dev. Biol. 305, 470-482.

Coutts, A. S., MacKenzie, E., Griffith, E. and Black, D. M. (2003). TES is a nove focal adhesion protein with a role in cell spreading. J. Cell Sci. 116, 897-906.

Delon, I. and Brown, N. H. (2009). The integrin adhesion complex changes its composition and function during morphogenesis of an epithelium. J. Cell Sci. 122 4363-4374

Deng, W.-M. and Ruohola-Baker, H. (2000). Laminin A is required for follicle celloocyte signaling that leads to establishment of the anterior-posterior axis in Drosophila. Curr. Biol. 10, 683-686.

Deng, W. M., Althauser, C. and Ruohola-Baker, H. (2001). Notch-Delta signaling induces a transition from mitotic cell cycle to endocycle in Drosophila follicle cells. Development 128, 4737-4746.

Deng, H., Wang, W., Yu, J., Zheng, Y., Qing, Y. and Pan, D. (2015). Spectrin regulates Hippo signaling by modulating cortical actomyosin activity. eLife $\mathbf{4}$ 1047.

Devenport, D. and Brown, N. H. (2004). Morphogenesis in the absence of integrins: mutation of both Drosophila beta subunits prevents midgut migration Development 131, 5405-5415.

Djiane, A., Yogev, S. and Mlodzik, M. (2005). The apical determinants aPKC and dPatj regulate Frizzled-dependent planar cell polarity in the Drosophila eye. Cell 121, 621-631.

Dobens, L. L. and Raftery, L. A. (2000). Integration of epithelial patterning and morphogenesis in Drosophila ovarian follicle cells. Dev. Dyn. 218, 80-93.

Dominguez-Gimenez, P., Brown, N. H. and Martin-Bermudo, M. D. (2007) Integrin-ECM interactions regulate the changes in cell shape driving the morphogenesis of the Drosophila wing epithelium. J. Cell Sci. 120, 1061-1071.

Dubreuil, R. R., Byers, T. J., Stewart, C. T. and Kiehart, D. P. (1990). A betaspectrin isoform from Drosophila (beta $\mathrm{H}$ ) is similar in size to vertebrate dystrophin J. Cell Biol. 111, 1849-1858.

Dubreuil, R. R., Frankel, J., Wang, P., Howrylak, J., Kappil, M. and Grushko, T. A. (1998). Mutations of alpha spectrin and labial block cuprophilic cell differentiation and acid secretion in the middle midgut of Drosophila larvae. Dev. Biol. 194, 1-11.

Dubreuil, R. R., Wang, P., Dahl, S., Lee, J. and Goldstein, L. S. (2000). Drosophila beta spectrin functions independently of alpha spectrin to polarize the $\mathrm{Na}, \mathrm{K}$ ATPase in epithelial cells. J. Cell Biol. 149, 647-656.

Dubreuil, R., Grushko, T., Baumann, O. (2001). Differential effects of a labia mutation on the development, structure, and function of stomach acid-secreting cells in Drosophila melanogaster larvae and adults. Cell Tissue Res. 306, 167-178

Featherstone, D. E., Davis, W. S., Dubreuil, R. R. and Broadie, K. (2001) Drosophila alpha- and beta-spectrin mutations disrupt presynaptic neurotransmitter release. J. Neurosci. 21, 4215-4224.

Fehon, R., Johansen, K., Rebay, I. and Artavanis-Tsakonas, S. (1991). Complex cellular and subcellular regulation of notch expression during embryonic and imaginal development of Drosophila: implications for notch function. J. Cell Biol. 113, 657-669.

Fehon, R. G., Dawson, I. A. and Artavanis-Tsakonas, S. (1994). A Drosophila homologue of membrane-skeleton protein 4.1 is associated with septate junctions and is encoded by the coracle gene. Development 120, 545-557.

Fernández-Miñán, A., Martín-Bermudo, M. D. and González-Reyes, A. (2007) Integrin signaling regulates spindle orientation in Drosophila to preserve the follicular-epithelium monolayer. Curr. Biol. 17, 683-688.
Fletcher, G. C., Elbediwy, A., Khanal, I., Ribeiro, P. S., Tapon, N. and Thompson, B. J. (2015). The Spectrin cytoskeleton regulates the Hippo signalling pathway. EMBO J. 34, 940-954.

Frydman, H. M. and Spradling, A. C. (2001). The receptor-like tyrosine phosphatase Lar is required for epithelial planar polarity and for axis determination within Drosophila ovarian follicles. Development 128, 3209.

Geiger, B., Spatz, J. P. and Bershadsky, A. D. (2009). Environmental sensing through focal adhesions. Nat. Rev. Mol. Cell Biol. 10, 21-33.

Gomez, J. M., Wang, Y. and Riechmann, V. (2012). Tao controls epithelial morphogenesis by promoting Fasciclin 2 endocytosis. J. Cell Biol. 199 1131-1143.

González-Reyes, A., Elliott, H. and St Johnston, D. (1995). Polarization of both major body axes in Drosophila by gurken-torpedo signalling. Nature 375, 654-658 He, L., Wang, X., Tang, H. L. and Montell, D. J. (2010). Tissue elongation requires oscillating contractions of a basal actomyosin network. Nat. Cell Biol. 12, 1133-1142.

Hildebrand, J. D. (2005). Shroom regulates epithelial cell shape via the apical positioning of an actomyosin network. J. Cell Sci. 118, 5191-5203.

Hulsmeier, J., Pielage, J., Rickert, C., Technau, G. M., Klambt, C. and Stork, T. (2007). Distinct functions of alpha-Spectrin and beta-Spectrin during axonal pathfinding. Development 134, 713-722.

Klebes, A. and Knust, E. (2000). A conserved motif in Crumbs is required for $E-$ cadherin localisation and zonula adherens formation in Drosophila. Curr. Biol. 10 76-85.

Kolahi, K. S., White, P. F., Shreter, D. M., Classen, A.-K., Bilder, D. and Mofrad M. R. K. (2009). Quantitative analysis of epithelial morphogenesis in Drosophila oogenesis: New insights based on morphometric analysis and mechanical modeling. Dev. Biol. 331, 129-139.

Laprise, P., Lau, K. M., Harris, K. P., Silva-Gagliardi, N. F., Paul, S. M., Beronja S., Beitel, G. J., McGlade, C. J. and Tepass, U. (2009). Yurt, Coracle, Neurexin IV and the $\mathrm{Na}(+), \mathrm{K}(+)$-ATPase form a novel group of epithelial polarity proteins. Nature 459, 1141-1145.

Lee, J, Coyne, R., Dubreuil, R. R., Goldstein, L. S. and Branton, D. (1993). Cell shape and interaction defects in alpha-spectrin mutants of Drosophila melanogaster. J. Cell Biol. 123, 1797-1809.

Lee, J. K., Brandin, E., Branton, D. and Goldstein, L. S. (1997). alpha-Spectrin is required for ovarian follicle monolayer integrity in Drosophila melanogaster. Development 124, 353-362.

MacDougall, N., Lad, Y., Wilkie, G. S., Francis-Lang, H., Sullivan, W. and Davis, I. (2001). Merlin, the Drosophila homologue of neurofibromatosis-2, is specifically required in posterior follicle cells for axis formation in the oocyte. Development 128, 665-673.

Mahowald, A. P. (1972). Ultrastructural observations on oogenesis in Drosophila. J. Morphol. 137, 29-48.

Martin, A. C., Kaschube, M. and Wieschaus, E. F. (2009). Pulsed contractions of an actin-myosin network drive apical constriction. Nature 457, 495-499.

Médina, E., Williams, J., Klipfell, E., Zarnescu, D., Thomas, G. and Le Bivic, A (2002). Crumbs interacts with moesin and beta(Heavy)-spectrin in the apical membrane skeleton of Drosophila. J. Cell Biol. 158, 941-951.

Meignin, C., Alvarez-Garcia, I., Davis, I. and Palacios, I. M. (2007). The salvadorwarts-hippo pathway is required for epithelial proliferation and axis specification in Drosophila. Curr. Biol. 17, 1871-1878.

Monier, B., Pélissier-Monier, A., Brand, A. H. and Sanson, B. (2010). An actomyosin-based barrier inhibits cell mixing at compartmental boundaries in Drosophila embryos. Nat. Cell Biol. 12, 60-65; sup pp 61-69.

Müller, H. A. (2000). Genetic control of epithelial cell polarity: lessons from Drosophila. Dev. Dyn. 218, 52-67.

Muzzopappa, M. and Wappner, P. (2005). Multiple roles of the F-box protein Slimb in Drosophila egg chamber development. Development 132, 2561-2571.

Patel, N. H., Snow, P. M. and Goodman, C. S. (1987). Characterization and cloning of fasciclin III: a glycoprotein expressed on a subset of neurons and axon pathways in Drosophila. Cell 48, 975-988.

Pesacreta, T. C., Byers, T. J., Dubreuil, R., Kiehart, D. P. and Branton, D. (1989) Drosophila spectrin: the membrane skeleton during embryogenesis. J. Cell Biol. 108, 1697-1709.

Pielage, J., Fetter, R. D. and Davis, G. W. (2005). Presynaptic spectrin is essential for synapse stabilization. Curr. Biol. 15, 918-928.

Polesello, C. and Tapon, N. (2007). Salvador-warts-hippo signaling promotes Drosophila posterior follicle cell maturation downstream of notch. Curr. Biol. 17, 1864-1870.

Ponceau, A., Albigès-Rizo, C., Colin-Aronovicz, Y., Destaing, O. and Lecomte, M.-C. (2015). alphall-spectrin regulates invadosome stability and extracellula matrix degradation. PLOS ONE 10, e0120781.

Roth, S., Neuman-Silberberg, F. S., Barcelo, G. and Schüpbach, T. (1995) cornichon and the EGF receptor signaling process are necessary for both anterior-posterior and dorsal-ventral pattern formation in Drosophila. Cell $\mathbf{8 1}$ 967-978.

Rotter, B., Bournier, O., Nicolas, G., Dhermy, D. and Lecomte, M.-C. (2005). Alphall-spectrin interacts with Tes and EVL, two actin-binding proteins located at cell contacts. Biochem. J. 388, 631-638. 
Royou, A., Field, C., Sisson, J., Sullivan, W. and Karess, R. (2004). Reassessing the role and dynamics of nonmuscle myosin II during furrow formation in early Drosophila embryos. Mol. Biol. Cell 15, 838-850.

Salomao, M., An, X., Guo, X., Gratzer, W. B., Mohandas, N. and Baines, A. J. (2006). Mammalian alpha I-spectrin is a neofunctionalized polypeptide adapted to small highly deformable erythrocytes. Proc. Natl. Acad. Sci. USA 103, 643-648.

Schaeffer, V., Althauser, C., Shcherbata, H. R., Deng, W.-M. and RuoholaBaker, H. (2004). Notch-dependent Fizzy-related/Hec1/Cdh1 expression is required for the mitotic-to-endocycle transition in Drosophila follicle cells. Curr. Biol. 14, 630-636

Sun, J. and Deng, W.-M. (2007). Hindsight mediates the role of notch in suppressing hedgehog signaling and cell proliferation. Dev. Cell 12, 431-442.

Suzuki, M., Morita, H. and Ueno, N. (2012). Molecular mechanisms of cell shape changes that contribute to vertebrate neural tube closure. Dev. Growth Differ. 54, 266-276.

Szafranski, P. and Goode, S. (2007). Basolateral junctions are sufficient to suppress epithelial invasion during Drosophila oogenesis. Dev. Dyn. 236 364-373.

Tepass, U. and Tanentzapf, G. (2001). Epithelial cell polarity and cell junctions in Drosophila. Annu. Rev. Genet. 35, 747-784.

Thomas, G. H. and Williams, J. A. (1999). Dynamic rearrangement of the spectrin membrane skeleton during the generation of epithelial polarity in Drosophila J. Cell Sci. 112, 2843-2852.

Thomas, G. H., Zarnescu, D. C., Juedes, A. E., Bales, M. A., Londergan, A., Korte, C. C. and Kiehart, D. P. (1998). Drosophila betaHeavy-spectrin is essential for development and contributes to specific cell fates in the eye. Development 125, 2125-2134.

Urwyler, O., Cortinas-Elizondo, F. and Suter, B. (2012). Drosophila sosie functions with $\beta(\mathrm{H})$-Spectrin and actin organizers in cell migration, epithelial morphogenesis and cortical stability. Biol. Open 1, 994-1005.

Wang, Y. and Riechmann, V. (2007). The role of the actomyosin cytoskeleton in coordination of tissue growth during Drosophila oogenesis. Curr. Biol. 17 1349-1355

Wei, J., Hortsch, M. and Goode, S. (2004). Neuroglian stabilizes epithelial structure during drosophila oogenesis. Dev. Dyn. 230, 800-808.

Wells, R. E., Barry, J. D., Warrington, S. J., Cuhlmann, S., Evans, P., Huber, W. Strutt, D. and Zeidler, M. P. (2013). Control of tissue morphology by Fasciclin IIImediated intercellular adhesion. Development 140, 3858-3868.
Widmann, T. J. and Dahmann, C. (2009). Dpp signaling promotes the cuboidal-tocolumnar shape transition of Drosophila wing disc epithelia by regulating Rho1. J. Cell Sci. 122, 1362-1373.

Williams, J. A., Maclver, B., Klipfell, E. A. and Thomas, G. H. (2004). The C-terminal domain of Drosophila (beta) heavy-spectrin exhibits autonomous membrane association and modulates membrane area. J. Cell Sci. 117, 771-782.

Williams, L. S., Ganguly, S., Loiseau, P., Ng, B. F. and Palacios, I. M. (2014). The auto-inhibitory domain and ATP-independent microtubule-binding region of Kinesin heavy chain are major functional domains for transport in the Drosophila germline. Development 141, 176-186.

Wodarz, A., Hinz, U., Engelbert, M. and Knust, E. (1995). Expression of crumbs confers apical character on plasma membrane domains of ectodermal epithelia of Drosophila. Cell 82, 67-76.

Wong, K. K. L., Li, W., An, Y., Duan, Y., Li, Z., Kang, Y. and Yan, Y. (2015). betaSpectrin Regulates the Hippo Signaling Pathway and Modulates the Basal Actin Network. J. Biol. Chem. 290, 6397-6407.

Woods, D. F. and Bryant, P. J. (1991). The discs-large tumor suppressor gene of Drosophila encodes a guanylate kinase homolog localized at septate junctions. Cell 66, 451-464

Woods, D. F. and Bryant, P. J. (1993). Apical junctions and cell signalling in epithelia. J. Cell Sci. Suppl. 17, 171-181.

Woods, D. F., Hough, C., Peel, D., Callaini, G. and Bryant, P. J. (1996). Dlg protein is required for junction structure, cell polarity, and proliferation control in Drosophila epithelia. J. Cell Biol. 134, 1469-1482

Wu, S., Huang, J., Dong, J. and Pan, D. (2003). hippo encodes a Ste-20 family protein kinase that restricts cell proliferation and promotes apoptosis in conjunction with salvador and warts. Cell 114, 445-456.

Xu, T. and Rubin, G. M. (1993). Analysis of genetic mosaics in developing and adult Drosophila tissues. Development 117, 1223-1237.

Yu, J., Poulton, J., Huang, Y.-C. and Deng, W.-M. (2008). The hippo pathway promotes Notch signaling in regulation of cell differentiation, proliferation, and oocyte polarity. PLOS ONE 3, e1761.

Zak, N. B. and Shilo, B.-Z. (1992). Localization of DER and the pattern of cell divisions in wild-type and Ellipse eye imaginal discs. Dev. Biol. 149, 448-456.

Zarnescu, D. and Thomas, G. (1999). Apical spectrin is essential for epithelial morphogenesis but not apicobasal polarity in Drosophila. J. Cell Biol. 146 1075-1086. 